\title{
Bound states to critical quasilinear Schrödinger equations
}

\author{
Youjun Wang and Wenming Zou
}

\begin{abstract}
In this paper, we consider the critical quasilinear Schrödinger equations of the form

$$
-\varepsilon^{2} \Delta u+V(x) u-\varepsilon^{2}\left[\Delta\left(u^{2}\right)\right] u=|u|^{2\left(2^{*}\right)-2} u+g(u), \quad x \in \mathbb{R}^{N},
$$

where $N \geq 3,2^{*}:=2 N /(N-2)$ and $g(u)$ is of subcritical growth. We prove the existence of positive bound states which concentrate around a local minimum point of $V$ as $\varepsilon \rightarrow 0^{+}$.

Mathematics Subject Classification (2000). 35B33, 35J20, 35J60, 35Q55.
\end{abstract}

\section{Introduction}

This paper is motivated by the recent interests on the following type of quasilinear Schrödinger equations

$i \varepsilon \frac{\partial \psi}{\partial t}=-\varepsilon^{2} \Delta \psi+W(x) \psi-\tilde{h}\left(|\psi|^{2}\right) \psi-\varepsilon^{2} k \Delta \tilde{\rho}\left(|\psi|^{2}\right) \tilde{\rho}^{\prime}\left(|\psi|^{2}\right) \psi, \quad x \in \mathbb{R}^{N}$,

where $W$ is a given potential, $k$ is a real constant, $\tilde{h}$ and $\tilde{\rho}$ are real functions of essentially pure power forms. Here we consider the case $\tilde{\rho}(s)=s$ and our special interest is the standing wave solutions, i.e., solutions of type $\psi(t, x)=\exp (-i E t) u(x)$, where $E \in \mathbb{R}$ and $u>0$ is a real function. Note that $\psi$ satisfies (1.1) if and only if the function $u(x)$ solves the following equation of elliptic type with the formal variational structure

$$
-\varepsilon^{2} \Delta u+V(x) u-\varepsilon^{2} k\left[\Delta\left(u^{2}\right)\right] u=h(u), \quad x \in \mathbb{R}^{N},
$$

where $V(x)=W(x)-E$ is the new potential function, $h$ is the new nonlinearity.

In quantum mechanics, where the number of particles is conserved, a bound state is a state in the Hilbert space that corresponds to two or more particles whose interaction energy determines whether these particles can be

This article was supported by NSFC (No. 10871109, 11025106) and China Post-Doc Science Foundation (No. 20100470175). 
separated or not. One of the historical puzzles that led to the creation of quantum mechanics was the stability of a bound state - why electrons in atoms do not just spiral into the nucleus. It is the bound states of nucleons, atoms, molecules and solids that allow the world and all of life to be what it is. Therefore, the bound state is one of the most important topics in quantum mechanics. So much current ongoing research and historical research using quantum mechanics involve the bound states.

The existence and concentration behavior of positive ground (bound) state solutions for (1.2) with $k=0$ has been studied extensively in recent years. Consider

$$
-\varepsilon^{2} \Delta u+V(x) u=f(x, u), \quad u>0, \quad u \in H^{1}\left(\mathbb{R}^{N}\right) .
$$

Note that when setting $v(x)=u(\varepsilon x)$ and $V_{\varepsilon}(x)=V(\varepsilon x)$, then (1.3) is equivalent to

$$
-\Delta v+V_{\varepsilon}(x) v=f(x, v), \quad v>0, \quad v \in H^{1}\left(\mathbb{R}^{N}\right) .
$$

For $\varepsilon$ small enough, the solutions to problem (1.3) can induce the standing waves of the Schrödinger equation which are usually referred to as semiclassical states. Some class of solutions of (1.3) concentrate and develop spike layers and peaks around certain points in $\mathbb{R}^{N}$ but vanishing elsewhere as $\varepsilon \rightarrow 0$. The existence of single-peak solutions was first studied in [21] where $N=1$ and $f=u^{3}$. A single-peak solution was constructed which concentrates around any given non-degenerate critical point of the potential $V(x)$. The higher dimension cases were considered in $[35,36]$. In particular, in [36], the existence of multi-peak solutions which concentrate around any finite subsets of the nondegenerate critical points of $V(x)$ was established. The arguments in $[21,35,36]$ are based on a Lyapunov-Schmidt reduction and heavily rely on the uniqueness and non-degeneracy of the positive ground state solutions (least energy solutions). In [2], they studied (1.3) and considered the concentration phenomena at isolated local minima and maxima with polynomial degeneracy. In [27], the author deals with $\mathbf{C}^{1}$-stable critical points of $V(x)$. See also $[3,12,25]$ for related results about (1.3). However, as observed by many experts, the uniqueness and non-degeneracy of the positive ground state solutions are usually quite difficult to verify. They are known so far only for some very restricted cases on nonlinearities $f$ in (1.3). To get the existence of positive solutions without these assumptions, the variational approach which was initiated in [40] has proved to be very successful. In [40], by the mountain pass theorem, the author proves the existence of positive solutions of (1.3) for small $\varepsilon>0$ whenever liminf $\left.\lim _{|x| \rightarrow \infty} V(x)\right)>\inf _{x \in \mathbb{R}^{N}} V(x)$. These solutions concentrate around the global minimum points of $V(x)$ when $\varepsilon \rightarrow 0$ as was shown in [42]. Later in [16], by introducing a penalization approach, the authors proved a localized version of the result in $[40,42]$, see also [13-15] for related results, they do not assume the uniqueness of a least-energy ground state in a related homogeneous problem. In $[9,24]$, the monotonicity condition of [16] is not necessary. Also, in [8], the authors develop a new variational approach to construct localized positive solutions to (1.3) which concentrate at an isolated component of positive 
local minimum points of $V(x)$ as $\varepsilon \rightarrow 0$ under certain conditions on $f$ which are "almost optimal". Similarly, no uniqueness and non-degeneracy of the positive ground state solutions are required in [8]. In [15,17], they constructed a family of solutions with several spikes located around any prescribed finite set of local minima of $V$. For more results of this type, we refer to [6-11,13].

Quasilinear Schrödinger equation of the type with $k>0$ arises in various fields of physics, like the theory of superfluids or dissipative quantum mechanics, see e.g. [26,33]. Equations with more general dissipative term arise in plasma physics, fluid mechanics, in the theory of Heisenberg ferromagnets, etc. For further physical motivations and a more complete list of references dealing with application, we refer the readers to [17-23,29] and their bibliography.

In [37], the existence of positive ground state solutions for the quasilinear Schrödinger equation $-u^{\prime \prime}+V(x) u-\left(u^{2}\right)^{\prime \prime} u=\theta|u|^{p-1} u$ in $\mathbb{R}$ were constructed as minimizers of a constrained minimization problem, with $\theta$ being the Lagrange multiplier. In [29], by a change of variables the quasilinear problem (1.2), where $h(x)$ is of subcritical growth, was transformed to a semilinear one and an Orlicz space framwork was used as the working space, and they were able to prove the existence of positive solutions by the mountain-pass Theorem. The same method of changing of variables was used in [10], but the usual Sobolev space $H^{1}\left(\mathbb{R}^{N}\right)$ framework was used as the working space and they proved the existence of a spherically symmetric solution from the classical results given by Berestycki and Lions [5].

The existence of solutions for problem (1.2) involving subcritical growth with $\varepsilon=1$ was considered in $[4,10,19,26,28,29]$ and it was left an open problem for the critical case in [29]. It seems that the existence of solutions for critical case was first studied by Moameni in [30] when the potential function $V$ satisfies some geometry conditions, and it was established the existence of multiple solutions in [31] by the fibering method. Recently, do Ó et al. [20] considered problem (1.2) with $\varepsilon=1, h(u)=|u|^{q-1} u+|u|^{p-1} u$, where $3<q<p \leq 2\left(2^{*}\right)-1$. They showed the existence of a positive classic solution if $V(x)$ is bounded or periodic. In [41], the authors study problem (1.2) with $\varepsilon=1, h(u)=K(x)|u|^{2\left(2^{*}\right)-1} u+g(x, u)$, where $g(x, u)$ is an asymptotically nonlinearity. They also proved one solution if $V(x)$ is periodic. There are few results dealing with semi-classical states for quasilinear Schrödinger equations of the form (1.2) with critical growth, which are families of solutions $u_{\varepsilon}$ which develop a spike shape around one or more distinguished points of the space, while vanishing asymptotically elsewhere as $\varepsilon \rightarrow 0$. We only refer to the recent paper [18] in the case of $N=2$.

Based on the above reviews and observations, we know that the existence of positive bound states along with the concentration behavior of solutions to the critical case of (1.2) with $N \geq 3$ is largely open.

The main purpose of the present paper is to show the existence and concentration behavior of positive bound state solutions for (1.2) when the 
nonlinearity has the critical nonlinearity. More precisely, we consider the problem

$$
-\varepsilon^{2} \Delta u+V(x) u-\varepsilon^{2}\left[\Delta\left(u^{2}\right)\right] u=|u|^{2\left(2^{*}\right)-2} u+g(u), \quad x \in \mathbb{R}^{N},
$$

where $N \geq 3,2^{*}=2 N /(N-2)$. As in [16], we also assume the following conditions on $V$ :

$\left(V_{1}\right)$ The function $V: \mathbb{R}^{N} \rightarrow \mathbb{R}$ is locally Hölder continuous and $V(x) \geq \alpha>0$ for all $x \in \mathbb{R}^{N}$.

$\left(V_{2}\right)$ There is a bounded domain $\Omega \subset \mathbb{R}^{N}$ such that $V_{0}:=\inf _{\Omega} V<\inf _{\partial \Omega} V$.

The nonlinear term $g: \mathbb{R}^{+} \rightarrow \mathbb{R}$ is of class $C^{1}$ and satisfies

$\left(g_{1}\right) g(s)=o(s)$ as $s \rightarrow 0$.

$\left(g_{2}\right)$ There are $q_{1}, q_{2} \in\left(2(N+2) /(N-2), 2\left(2^{*}\right)\right)$ and a $\lambda \in(0,+\infty)$ such that

$$
g(s) \geq \lambda s^{q_{1}-1} \quad \text { for all } s>0 \text { and } \lim _{s \rightarrow \infty} \frac{g(s)}{s^{q_{2}-1}}=0 .
$$

$\left(g_{3}\right)$ There exists $\theta \in\left(4, q_{2}\right)$ such that

$$
0<G(s)=\int_{0}^{s} g(t) d t \leq \frac{1}{\theta} g(s) s \text { for all } s>0 .
$$

$\left(g_{4}\right)$ The function $g(s) s^{-3}$ is increasing for $s>0$.

Our main result in the current paper is the following

Theorem 1.1. Suppose that $\left(V_{1}\right)-\left(V_{2}\right)$ and $\left(g_{1}\right)-\left(g_{4}\right)$ hold. Then there is an $\varepsilon_{0}>0$ such that problem (1.4) possesses a positive bound state solution $u_{\varepsilon} \in$ $C_{\text {loc }}^{2, \alpha}\left(\mathbb{R}^{N}\right)$ for all $0<\varepsilon<\varepsilon_{0}$ and some $\alpha \in(0,1)$. Moreover, $u_{\varepsilon}$ has at most one local (global) maximum $z_{\varepsilon}$ in $\mathbb{R}^{N}$, which is inside $\Omega$ such that

$$
\lim _{\varepsilon \rightarrow 0^{+}} V\left(z_{\varepsilon}\right)=V_{0}:=\inf _{\Omega} V .
$$

Besides, there exist $C, \xi>0$ such that

$$
u_{\varepsilon}(z) \leq C \exp \left(-\xi\left|\frac{z-z_{\varepsilon}}{\varepsilon}\right|\right) .
$$

\section{Reformulation of the problem}

It should be pointed out that we may not apply directly the variational method to study (1.4) since the natural associated functional $\tilde{I}$ given by

$$
\begin{aligned}
\tilde{I}(u)= & \frac{1}{2} \varepsilon^{2} \int_{\mathbb{R}^{N}}\left(1+2 u^{2}\right)|\nabla u|^{2} d x+\frac{1}{2} \int_{\mathbb{R}^{N}} V(x) u^{2} d x-\frac{1}{2\left(2^{*}\right)} \int_{\mathbb{R}^{N}} u^{2\left(2^{*}\right)} d x \\
& -\int_{\mathbb{R}^{N}} G(u) d x
\end{aligned}
$$

is not well defined in general, for instance, in $H^{1}\left(\mathbb{R}^{N}\right)$. To overcome this difficulty, we employ an argument developed by Colin and Jeanjean [10] (see also $[19,29])$. We make the changing of variables $v=f^{-1}(u)$, where $f$ is defined by: $f^{\prime}(t)=\frac{1}{\sqrt{1+2 f^{2}(t)}}$ on $[0,+\infty)$ and $f(t)=-f(-t)$ on $(-\infty, 0]$. 


\section{Lemma 2.1. We have that}

(i) the functional $f(t) f^{\prime}(t) t^{-1}$ is decreasing for $t>0$;

(ii) the functional $f^{q}(t) f^{\prime}(t) t^{-1}$ is increasing for $q \geq 3$ and $t>0$.

Proof. (i) was proved in [18]. To prove $(i i)$, by the definition of $f$, we have $f(t) / 2 \leq t f^{\prime}(t) \leq f(t)$ for all $t \geq 0$ and $\left|f(t) f^{\prime}(t)\right| \leq 1 / \sqrt{2}$ for all $t \in \mathbb{R}$ (see [19]). Then,

$$
\begin{aligned}
\frac{d}{d t}\left[\frac{f^{q}(t) f^{\prime}(t)}{t}\right] & =\frac{q f^{q-1}(t)\left(f^{\prime}(t)\right)^{2} t-2 f^{q+1}(t)\left(f^{\prime}(t)\right)^{4} t-f^{q}(t) f^{\prime}(t)}{t^{2}} \\
& \geq \frac{f^{q-1}(t) f^{\prime}(t)\left[q f^{\prime}(t) t-2 f^{2}(t)\left(f^{\prime}(t)\right)^{3} t-f(t)\right]}{t^{2}} \\
& \geq \frac{f^{q-1}(t) f^{\prime}(t)\left[(q-1) f^{\prime}(t) t-f(t)\right]}{t^{2}} \\
& >0 .
\end{aligned}
$$

The lemma is proved.

After the change of variables $(u=f(v))$, we can rewrite $\tilde{I}(u)$ as

$$
\begin{aligned}
\tilde{J}(v)= & \frac{1}{2} \int_{\mathbb{R}^{N}}\left(\varepsilon^{2}|\nabla v|^{2}+V(x) f^{2}(v)\right) d x-\frac{1}{2\left(2^{*}\right)} \int_{\mathbb{R}^{N}}|f(v)|^{2\left(2^{*}\right)-2} f(v) d x \\
& -\int_{\mathbb{R}^{N}} G(f(v)) d x .
\end{aligned}
$$

Then, the critical points of $\tilde{J}$ are weak solutions of the equation

$$
-\varepsilon^{2} \Delta v=f^{\prime}(v)\left(g(f(v))+|f(v)|^{2\left(2^{*}\right)-2} f(v)-V(x) f(v)\right), \quad x \in \mathbb{R}^{N} .
$$

Note that if $v$ is a critical point of $\tilde{J}(v)$, then, $u=f(v)$ is a weak solution of the equation (1.4). As in [19], we define the Orlicz space

$$
E:=\left\{v \in H^{1}\left(\mathbb{R}^{N}\right): \int_{\mathbb{R}^{N}} V(x) f^{2}(v) d x<\infty\right\}
$$

which is endowed with the norm $\|v\|=\|\nabla v\|_{2}+\inf _{\xi>0} \frac{1}{\xi}\left[1+\int_{\mathbb{R}^{N}} V(x) f^{2}(\xi v) d x\right]$. Let $X:=\left\{u \in H^{1}\left(\mathbb{R}^{N}\right): \int_{\mathbb{R}^{N}} V(x) u^{2} d x<\infty\right\}$, which is endowed with the inner product $\langle u, v\rangle=\int_{\mathbb{R}^{N}}(\nabla u \nabla v+V(x) u v) d x$ and the corresponding norm

$$
\|u\|_{X}=\left(\int_{\mathbb{R}^{N}}\left(|\nabla u|^{2}+V(x) u^{2}\right) d x\right)^{1 / 2} .
$$


Proposition 2.1. The functional $\tilde{J}$ has the following properties:

(1) $\tilde{J}$ is well defined in $E$.

(2) $\tilde{J}$ is continuous in $E$.

(3) $\tilde{J}$ is Gateaux-differentiable in E.

Proof. The proof is similar to the proof of Proposition 2.5 in [30].

The following result can be found in [19].

\section{Proposition 2.2.}

(1) E is a Banach space.

(2) There exists a $C>0$ such that

$$
\frac{\int_{\mathbb{R}^{N}} V(x) f^{2}(v) d x}{1+\left(\int_{\mathbb{R}^{N}} V(x) f^{2}(v) d x\right)^{1 / 2}} \leq C\|v\|, \quad \forall v \in E .
$$

(3) The embedding $X \hookrightarrow E$ is continuous.

(4) The map $v \rightarrow f(v)$ from $E$ to $L^{q}\left(\mathbb{R}^{N}\right)$ is continuous for $2 \leq q \leq 2\left(2^{*}\right)$.

(5) The embedding $E \hookrightarrow L^{r}\left(\mathbb{R}^{N}\right)$ is continuous for $2 \leq r \leq 2\left(2^{*}\right)$.

(6) The embedding $E \hookrightarrow H^{1}\left(\mathbb{R}^{N}\right)$ is continuous. Moreover, $C_{0}^{\infty}\left(\mathbb{R}^{N}\right)$ is dense in $E$.

Before closing this section, we establish the following lemma.

Lemma 2.2. Let $\left\{v_{n}\right\}$ be a bounded sequence in $E$ such that

$$
\liminf _{n \rightarrow \infty} \sup _{y \in \mathbb{R}^{N}} \int_{B_{R}(y)} f^{2}\left(v_{n}\right) d x \rightarrow 0
$$

for some $R>0$. Then $f\left(v_{n}\right) \rightarrow 0$ in $L^{s}\left(\mathbb{R}^{N}\right)$ for $2<s<2\left(2^{*}\right)$.

Proof. Since $E \hookrightarrow H^{1}(\mathbb{R})$ is continuous, $\left\{v_{n}\right\}$ is also bounded in $H^{1}\left(\mathbb{R}^{N}\right)$. It follows from the Hölder and Sobolev inequalities, we have

$$
\begin{aligned}
\int_{B_{R}(y)}|f(v)|^{s} d x & \leq\left(\int_{B_{R}(y)} f^{2}(v) d x\right)^{\frac{(1-\alpha) s}{2}}\left(\int_{B_{R}(y)}\left(f^{2}(v)\right)^{2^{*}} d x\right)^{\frac{\alpha s}{2\left(2^{*}\right)}} \\
& \leq C\left(\int_{B_{R}(y)} f^{2}(v) d x\right)^{\frac{(1-\alpha) s}{2}}\left(\int_{B_{R}(y)}\left(|\nabla v|^{2}+v^{2}\right) d x\right)^{\frac{\alpha s}{4}}
\end{aligned}
$$

where $\alpha=\frac{2^{*} s-2\left(2^{*}\right)}{\left(2^{*}-1\right) s}$. If $s \geq \frac{4}{N}$, then $\alpha s \geq 4$ and

$\int_{B_{R}(y)}|f(v)|^{s} d x \leq C\left(\int_{B_{R}(y)} f^{2}(v) d x\right)^{\frac{(1-\alpha) s}{2}}\|v\|_{H^{1}}^{\frac{\alpha s-4}{2}} \int_{B_{R}(y)}\left(|\nabla v|^{2}+v^{2}\right) d x$.

Covering $\mathbb{R}^{N}$ by a family of balls $\left\{B_{R}\left(y_{i}\right)\right\}$ such that each point is contained in at most $k$ such balls and summing up these inequalities over this family of balls we obtain

$$
\int_{\mathbb{R}^{N}}|f(v)|^{s} d x \leq k C \sup _{y \in \mathbb{R}^{N}}\left(\int_{B_{R}(y)} f^{2}(v) d x\right)^{\frac{(1-\alpha) s}{2}}\|v\|_{H^{1}}^{\frac{\alpha s}{2}}
$$


Substituting $v=v_{n}$ the result follows for $s \geq \frac{4}{N}$. If $2<s<\frac{4}{N}$, we write $s=2 \tau+(1-\tau) \frac{4}{N}$ for some $\tau \in(0,1)$. By the Hölder inequality, we get

$$
\|f(v)\|_{s}^{s} \leq\|f(v)\|_{2}^{2 \tau}\|f(v)\|_{\frac{4}{N}}^{(1-\tau) \frac{4}{N}}
$$

and the result in this case follows the conclusion already established above.

\subsection{Penalized nonlinearity}

We consider the following Carathéodory function:

$$
h(x, s)= \begin{cases}\chi_{\Omega}\left(g(s)+s^{2\left(2^{*}\right)-1}\right)+\left(1-\chi_{\Omega}\right) \tilde{g}(s) & \text { if } s \geq 0 \\ 0 & \text { if } s<0\end{cases}
$$

where

$$
\tilde{g}(s)= \begin{cases}g(s)+s^{2\left(2^{*}\right)-1} & \text { if } s \leq a \\ k^{-1} \alpha s & \text { if } s>a\end{cases}
$$

with $k>\frac{2(\theta-2)}{\theta-4}>2, a>0$ such that $g(a)+a^{2\left(2^{*}\right)-1}=k^{-1} a \alpha, \chi_{\Omega}$ is the characteristics function on $\Omega$.

Using $\left(g_{1}\right)-\left(g_{4}\right)$, it is easy to check that $h(x, s)$ satisfies the following conditions:

$\left(h_{1}\right) h(x, s)=o(s)$ as $s \rightarrow 0$;

$\left(h_{2}\right) h(x, s) \leq g(s)+s^{2\left(2^{*}\right)-1}$ for all $s>0, x \in \mathbb{R}^{N}$;

$\left(h_{3}\right) 0<\theta H(x, s) \leq h(x, s) s$ for all $x \in \Omega, s>0$ or $x \notin \Omega, s \leq a ; 0 \leq$ $2 H(x, s) \leq h(x, s) s \leq k^{-1} V(x) s^{2}$ for all $x \notin \Omega, s>0$, where $H(x, s)=$ $\int_{0}^{s} h(x, t) d t$

$\left(h_{4}\right)$ The function $h(x, s) s^{-3}$ is increasing for $s>0$.

We introduce the penalized functional $J: E \rightarrow \mathbb{R}$ as following

$$
J(v)=\frac{1}{2} \int_{\mathbb{R}^{N}}\left(\varepsilon^{2}|\nabla v|^{2}+V(x) f^{2}(v)\right) d x-\int_{\mathbb{R}^{N}} H(x, f(v)) d x .
$$

Using Proposition 2.2, it is easy to check that $J$ is well defined and that $J \in C^{1}(E, \mathbb{R})$. Moreover, the critical points of $J$ are solutions of the modified problem

$$
-\varepsilon^{2} \Delta v=f^{\prime}(v)(h(x, f(v))-V(x) f(v)), \quad x \in \mathbb{R}^{N} .
$$

Next, we assume that $\varepsilon=1$.

\subsection{Mountain pass geometry}

Consider the set $S(\rho)=\left\{v \in E: \Phi(v)=\rho^{2}\right\}$, where $\Phi: E \rightarrow \mathbb{R}$ is given by

$$
\Phi(v)=\int_{\mathbb{R}^{N}}\left(|\nabla v|^{2}+V(x) f^{2}(v)\right) d x .
$$

Since $\Phi(v)$ is continuous then $S(\rho)$ is a closed subset and disconnects the space $E$ for $\rho>0$. 
Lemma 2.3. There exist $\rho_{0}, a_{0}>0$ such that $J(v) \geq a_{0}$ for all $v \in S\left(\rho_{0}\right)$.

Proof. For $v \in S(\rho)$, by the Hölder and the Sobolev inequalities, we have

$$
\begin{aligned}
\int_{\mathbb{R}^{N}}|f(v)|^{q_{2}} d x & \leq\left(\int_{\mathbb{R}^{N}} f^{2}(v) d x\right)^{\frac{r q_{2}}{2}}\left(\int_{\mathbb{R}^{N}} f^{2\left(2^{*}\right)}(v) d x\right)^{1-\frac{r q_{2}}{2}} \\
& \leq C\left(\rho^{2}\right)^{\frac{r q_{2}}{2}}\left(\int_{\mathbb{R}^{N}}\left|\nabla f^{2}(v)\right|^{2} d x\right)^{\frac{\left(1-r q_{2} / 2\right) 2^{*}}{2}} \\
& \leq C\left(\rho^{2}\right)^{\frac{r q_{2}}{2}}\left(\int_{\mathbb{R}^{N}}|\nabla v|^{2} d x\right)^{\frac{\left(1-r q_{2} / 2\right) 2^{*}}{2}} \\
& \leq C \rho^{\frac{2 N+2 q_{2}}{N+2}}
\end{aligned}
$$

where $r=\frac{2\left(2^{*}\right)-q_{2}}{q_{2}\left(2^{*}-1\right)}$. Therefore, by $\left(h_{2}\right),\left(g_{1}\right)$ and $\left(g_{2}\right)$, we have

$$
\begin{aligned}
J(v)= & \frac{1}{2} \int_{\mathbb{R}^{N}}\left(|\nabla v|^{2}+V(x) f^{2}(v)\right) d x-\int_{\mathbb{R}^{N}} H(x, f(v)) d x \\
\geq & \frac{1}{2} \int_{\mathbb{R}^{N}}\left(|\nabla v|^{2}+V(x) f^{2}(v)\right) d x-\int_{\mathbb{R}^{N}}\left(G(f(v))+\frac{1}{2\left(2^{*}\right)} f^{2\left(2^{*}\right)}(v)\right) d x \\
\geq & \frac{1}{2} \int_{\mathbb{R}^{N}}\left(|\nabla v|^{2}+V(x) f^{2}(v)\right) d x \\
& -\int_{\mathbb{R}^{N}}\left(\epsilon f^{2}(v)+C \epsilon|f(v)|^{q_{2}}+\frac{1}{2\left(2^{*}\right)} f^{2\left(2^{*}\right)}(v)\right) d x \\
\geq & \frac{1}{4} \rho^{2}-C \rho^{\frac{2 N+2 q_{2}}{N+2}}-C \rho^{2^{*}}, \quad \text { for } \epsilon \text { small enough. }
\end{aligned}
$$

Since $\left(2 N+2 q_{2}\right) /(N+2)>2$, we choose $\rho_{0}>0$ small enough such that

$$
\beta_{0}:=\frac{1}{4}-C \rho^{\frac{2\left(q_{2}-2\right)}{N+2}}-C \rho^{\frac{4}{N-2}}>0,
$$

we have $J(v) \geq a_{0}:=\rho_{0}^{2} \beta_{0}>0$ for all $v \in S\left(\rho_{0}\right)$. 
Lemma 2.4. There exists an $v \in E$ such that $\Phi(v)>\rho_{0}^{2}$ and $J(v)<0$.

Proof. Given a $\varphi \in C_{0}^{\infty}\left(\mathbb{R}^{N},[0,1]\right)$ with $\operatorname{supp} \varphi=\bar{B}_{1}$, we prove that $J(t \varphi) \rightarrow$ $-\infty$ as $t \rightarrow \infty$, which proves our thesis if we take $v=t \varphi$ with $t$ large enough. Since $t f^{\prime}(t) \leq f(t)$, it follows that the function $f(t) / t$ is decreasing for $t>0$. Since $0<t \varphi(x) \leq t$ for $x \in B_{1}$, we have $f(t \varphi(x)) \geq f(t) \varphi(x)$, which implies that

$$
\begin{aligned}
J(t \varphi) & \leq \frac{1}{2} \int_{B_{1}}\left(|\nabla(t \varphi)|^{2}+V(x) f^{2}(t \varphi)\right) d x-\frac{1}{2\left(2^{*}\right)} \int_{B_{1}} f^{2\left(2^{*}\right)}(t \varphi) d x \\
& \leq \frac{t^{2}}{2} \int_{B_{1}}\left(|\nabla \varphi|^{2}+V(x) \varphi^{2}\right) d x-\frac{1}{2\left(2^{*}\right)} \int_{B_{1}} f^{2\left(2^{*}\right)}(t \varphi) d x \\
& \leq t^{2}\left[\frac{1}{2} \int_{B_{1}}\left(|\nabla \varphi|^{2}+V(x) \varphi^{2}\right) d x-\frac{f^{2\left(2^{*}\right)}(t)}{2\left(2^{*}\right) t^{2}} \int_{B_{1}} \varphi^{2\left(2^{*}\right)} d x\right] \\
& \rightarrow-\infty, \quad \text { as } t \rightarrow \infty
\end{aligned}
$$

since $\lim _{t \rightarrow+\infty} \frac{f^{2\left(2^{*}\right)}(t)}{t^{2}}=+\infty$.

Now, in view of Lemmas 2.3, 2.4, we can apply a version of mountain pass Theorem without $(\mathrm{PS})_{c}$ condition due to Ambrosetti-Rabinowitz [39], it follows that there exists a $(\mathrm{PS})_{c}$ sequence $\left\{v_{n}\right\} \subset E$, i.e., a sequence such that $J\left(v_{n}\right) \rightarrow c$ and $J^{\prime}\left(v_{n}\right) \rightarrow 0$, where $c$ is the mountain pass level of $J$ characterized by

$$
c=\inf _{\gamma \in \Gamma} \sup _{t \in[0,1]} J(\gamma(t))
$$

where $\Gamma=\{\gamma \in C([0,1], E): \gamma(0)=0, J(\gamma(1))<0, \gamma(1) \neq 0\}$. Let us consider the Nehari manifold $\mathcal{N}=\left\{v \in E \backslash\{0\}:\left\langle J^{\prime}(v), v\right\rangle=0\right\}$. We have the following lemma.

Lemma 2.5. For every $v \in E \backslash\{0\}$, there exists a unique $t_{0}>0$ such that $t_{0} v \in \mathcal{N}$. Moreover, $J\left(t_{0} v\right)=\max _{t \geq 0} J(t v)$.

Proof. Let $v \in E \backslash\{0\}$ be fixed and define the function $\mathcal{I}(t)=J(t|v|)$ for $t \geq 0$. We notice that $\mathcal{I}^{\prime}(t)=\left\langle J^{\prime}(t|v|),|v|\right\rangle=0$ if and only if $t v \in \mathcal{N}$. Moreover, $\mathcal{I}^{\prime}(t)=0$ is equivalent to

$$
\int_{\mathbb{R}^{N}}|\nabla v|^{2} d x=\int_{\mathbb{R}^{N}}\left[\frac{h(x, f(t|v|)) f^{\prime}(t|v|)}{t|v|}-\frac{V(x) f(t|v|) f^{\prime}(t|v|)}{t|v|}\right] v^{2} d x
$$

The right hand side of Eq. (2.4) is an increasing function of $t$. Indeed, for $x$ fixed, we consider the function $z:(0, \infty) \rightarrow \mathbb{R}$ given by

$$
z(s)=\frac{h(x, f(s)) f^{\prime}(s)}{s}-\frac{V(x) f(s) f^{\prime}(s)}{s} .
$$


If $x \in \Omega$, we have

$$
z(s)=\frac{\left[g(f(s))+f^{2\left(2^{*}\right)-1}(s)\right]}{f^{3}(s)} \frac{f^{3}(s) f^{\prime}(s)}{s}-\frac{V(x) f(s) f^{\prime}(s)}{s} .
$$

If $x \notin \Omega$, we have

$$
z(s)=\frac{\left[g(f(s))+f^{2\left(2^{*}\right)}(s)\right]}{f^{3}(s)} \frac{f^{3}(s) f^{\prime}(s)}{s}-\frac{V(x) f(s) f^{\prime}(s)}{s}, \quad s \leq f^{-1}(a),
$$

and

$$
z(s)=\frac{k V(x)-\alpha}{k}\left(-\frac{f(s) f^{\prime}(s)}{s}\right), \quad s>f^{-1}(a) .
$$

Thus, Lemma 2.1 and $\left(g_{4}\right)$ imply the claim. Since $v$ is fixed, we may choose $\delta>0$ such that

$$
\int_{\mathbb{R}^{N}}|\nabla v|^{2} d x-\delta \int_{\mathbb{R}^{N}}|v|^{2} d x>0 .
$$

By the definition of $h(x, s),\left(h_{2}\right),\left(g_{1}\right),\left(g_{2}\right)$ and $\left(f_{2}\right)$, we have

$$
\begin{aligned}
\lim _{t \rightarrow 0^{+}} \int_{\mathbb{R}^{N}} \frac{h(x, f(t v)) f(t v)}{t^{2}} d x \leq & \delta \lim _{t \rightarrow 0^{+}} \int_{\mathbb{R}^{N}} \frac{f^{2}(t v)}{t^{2}} d x \\
& +C \lim _{t \rightarrow 0^{+}} \int_{\mathbb{R}^{N}} \frac{f^{2(2 *)}(t v)}{t^{2}} d x \\
\leq & \delta \int_{\mathbb{R}^{N}} v^{2} d x+C \lim _{t \rightarrow 0^{+}} t^{2\left(2^{*}\right)-2} \int_{\mathbb{R}^{N}} v^{2\left(2^{*}\right)} d x \\
= & \delta \int_{\mathbb{R}^{N}} v^{2} d x
\end{aligned}
$$

By $\left(h_{3}\right)$, we have

$$
\begin{aligned}
\mathcal{I}(t) & =t^{2}\left(\frac{1}{2} \int_{\mathbb{R}^{N}}|\nabla v|^{2} d x+\frac{1}{2} \int_{\mathbb{R}^{N}} \frac{V(x) f^{2}(t v)}{t^{2}} d x-\int_{\mathbb{R}^{N}} \frac{H(x, f(t v))}{t^{2}} d x\right) \\
& \geq \frac{1}{2} t^{2}\left(\int_{\mathbb{R}^{N}}|\nabla v|^{2} d x-\int_{\mathbb{R}^{N}} \frac{h(x, f(t v)) f(t v)}{t^{2}} d x\right) .
\end{aligned}
$$

It follows by (2.5) and (2.6) that $\mathcal{I}(t)>0$ for small $t>0$. Moreover, $\mathcal{I}(0)=0$ and $\mathcal{I}(t)<0$ for $t$ large. Therefore, there exists a unique $t_{0}>0$ such that $\mathcal{I}^{\prime}\left(t_{0}\right)=0$, that is, $t_{0} v \in \mathcal{N}$. Furthermore, $\mathcal{I}\left(t_{0}\right)=\max _{t \geq 0} \mathcal{I}(t)$.

Define

$$
c^{*}=\inf _{v \in \mathcal{N}} J(v), \quad c^{* *}=\inf _{v \in E \backslash\{0\}} \max _{t \geq 0} J(t v) .
$$


Lemma 2.6. $c=c^{*}=c^{* *}$.

Proof. This kind of result has already been studied for Eq. (1.2) in [16,40,43] with $k=0$ and [18] with $k \neq 0$. We sketch the proof as following. It follows from Lemma 2.4 that $c^{*}=c^{* *}$. Note $J\left(t_{0} v\right)<0$ for $v \in E \backslash\{0\}$ and $t_{0}$ large enough. Defining $\gamma:[0,1] \rightarrow E$ by $\gamma(t)=t t_{0} v$, it follows that $\gamma \in \Gamma$ and consequently, $c \leq c^{* *}$. Next, we show that $c^{*} \leq c$. The manifold $\mathcal{N}$ separates $E$ into two components. As in the proof of Lemma 2.3, it follows that

$$
\begin{aligned}
\left\langle J^{\prime}(v), v\right\rangle= & \int_{\mathbb{R}^{N}}\left(|\nabla v|^{2}+V(x) f(v) f^{\prime}(v) v^{2}\right) d x-\int_{\mathbb{R}^{N}} h(x, f(v)) f^{\prime}(v) v d x \\
\geq & \frac{1}{4} \int_{\mathbb{R}^{N}}\left(|\nabla v|^{2}+V(x) f(v) f^{\prime}(v) v^{2}\right) d x \\
& -C\left(\int_{\mathbb{R}^{N}}\left(|\nabla v|^{2}+V(x) f(v) f^{\prime}(v) v^{2}\right) d x\right)^{\frac{N+q_{2}}{N+2}} \\
& -C\left(\int_{\mathbb{R}^{N}}\left(|\nabla v|^{2}+V(x) f(v) f^{\prime}(v) v^{2}\right) d x\right)^{\frac{2^{*}}{2}} .
\end{aligned}
$$

Using (2.1), notice that if $\|v\| \rightarrow 0$ then $\int_{\mathbb{R}^{2}}\left(|\nabla v|^{2}+V(x) f^{2}(v)\right) d x \rightarrow 0$, which implies that there exists a $\delta>0$ such that $\left\langle J^{\prime}(v), v\right\rangle>0$ when $0<\|v\|<\delta$. This proves that the component including the origin also contains a small ball around the origin. Moreover, $J(v) \geq 0$ for all $v$ in this component, because $\left\langle J^{\prime}(t v), v\right\rangle>0$ for all $0 \leq t \leq t_{0}$. Thus, $\gamma(0)=0$ and $\gamma(1)$ are in different components, which shows that every path $\gamma \in \Gamma$ has to cross $\mathcal{N}$. Therefore, we must have $c^{* *} \leq c$ and consequently $c^{*} \leq c$. The lemma is proved.

\subsection{Estimates for the minimax Level}

Proposition 2.3. There exits a $\vartheta \in E \backslash\{0\}$ such that $\max _{t>0} J(t \vartheta)<\frac{1}{2 N} S^{N / 2}$. Therefore, the mountain pass value c defined in (2.3) satisfies $c<\frac{1}{2 N} S^{N / 2}$, where $S$ is the best constant for the embedding $D^{1,2}\left(\mathbb{R}^{N}\right) \hookrightarrow L^{2^{*}}\left(\mathbb{R}^{N}\right)$.

Proof. Consider the functional $I: \tilde{E} \rightarrow \mathbb{R}$ given by

$$
I(u)=\frac{1}{2} \int_{\mathbb{R}^{N}}\left(1+2 u^{2}\right)|\nabla u|^{2} d x+\frac{1}{2} \int_{\mathbb{R}^{N}} V(x) u^{2} d x-\int_{\mathbb{R}^{N}} H(x, u) d x
$$

where $\tilde{E}:=\left\{u \in X: \int_{\mathbb{R}^{N}}\left|\nabla u^{2}\right|^{2} d x<\infty\right\}$. Then, by Proposition 2.2 (3), it suffices to show that there is $0 \neq \vartheta \in \tilde{E}$ such that

$$
\sup _{t \geq 0} I(t \vartheta)<\frac{1}{2 N} S^{N / 2}
$$

Indeed, since $J\left(f^{-1}(t \vartheta)\right)=-\infty$ as $t \rightarrow \infty$, there exists a $t_{0} \neq 0$ such that $J\left(f^{-1}\left(t_{0} \vartheta\right)\right)<0$. Set $\gamma_{1}(t):=f^{-1}\left(t t_{0} \vartheta\right)$. It follows from the definition of the mountain pass value that 


$$
\begin{aligned}
c & =\inf _{\gamma \in \Gamma} \sup _{t \in[0,1]} J(\gamma(t)) \leq \sup _{t \in[0,1]} J\left(\gamma_{1}(t)\right)=\sup _{t \in[0,1]} J\left(f^{-1}\left(t t_{0} \vartheta\right)\right) \\
& \leq \sup _{t \geq 0} I(t \vartheta)<\frac{1}{2 N} S^{N / 2} .
\end{aligned}
$$

Let $R>0$ be fixed and $\varphi \in C_{0}^{\infty}\left(\mathbb{R}^{N}\right)$ be a cut-off function with $\operatorname{supp} \varphi(x) \subset$ $B_{2 R} \subset \Omega$ such that $\varphi=1$ if $x \in B_{R}$ and $\varphi(x)=0$ for $x \in B_{2 R}^{c} ; 0 \leq \varphi(x) \leq 1$ for all $x$. It is known that the function

$$
U_{\varepsilon}=\frac{[N(N-2) \varepsilon]^{(N-2) / 4}}{\left(\varepsilon+|x|^{2}\right)^{(N-2) / 2}}, \quad \forall \varepsilon>0
$$

satisfies the equation $-\Delta u=|u|^{2^{*}-2} u$ in $\mathbb{R}^{N}$. We now consider the following function

$$
w_{\varepsilon}:=\varphi(x) U_{\varepsilon}^{1 / 2}=\frac{\varphi(x)[N(N-2) \varepsilon]^{(N-2) / 8}}{\left(\varepsilon+|x|^{2}\right)^{(N-2) / 4}}, \quad \forall \varepsilon>0 .
$$

From the definition of $w_{\varepsilon}$, we have

$$
\int_{B_{R}}\left|\nabla w_{\varepsilon}^{2}\right|^{2} d x \leq \int_{B_{R}}\left|w_{\varepsilon}^{2}\right|^{2^{*}} d x, \quad \int_{B_{R}^{c}}\left|w_{\varepsilon}^{2}\right|^{2^{*}} d x=O\left(\varepsilon^{(N-2) / 2}\right), \quad \text { as } \varepsilon \rightarrow 0 .
$$

Using the similar arguments as that in Brezis and Nirenberg [6], if we let $\vartheta_{\varepsilon}=\frac{w_{\varepsilon}}{\left\|w_{\varepsilon}^{2}\right\|_{2^{*}}^{\frac{1}{2}}}$, then we have

$$
\left\|\nabla \vartheta_{\varepsilon}^{2}\right\|_{2}^{2} \leq S+O\left(\varepsilon^{(N-2) / 2}\right) .
$$

On the other hand, $\lim _{t \rightarrow \infty} I\left(t \vartheta_{\varepsilon}\right)=-\infty$, which implies that there exists a $t_{\varepsilon} \geq 0$ such that $\sup _{t \geq 0} I\left(t \vartheta_{\varepsilon}\right)=I\left(t_{\varepsilon} \vartheta_{\varepsilon}\right)$. By $\left(g_{2}\right)$, we have

$$
\begin{aligned}
I\left(t \vartheta_{\varepsilon}\right) \leq & \frac{t^{2}}{2} \int_{\mathbb{R}^{N}}\left(\left|\nabla \vartheta_{\varepsilon}\right|^{2}+\|V\|_{L^{\infty}(\Omega)} \vartheta_{\varepsilon}^{2}\right) d x+\frac{t^{4}}{4} \int_{\mathbb{R}^{N}}\left|\nabla \vartheta_{\varepsilon}^{2}\right|^{2} d x \\
& -\frac{\lambda t^{q_{1}}}{q_{1}} \int_{\mathbb{R}^{N}}\left|\vartheta_{\varepsilon}\right|^{q_{1}} d x-\frac{t^{2\left(2^{*}\right)}}{2\left(2^{*}\right)},
\end{aligned}
$$

then $t_{\varepsilon}$ satisfies

$$
\begin{aligned}
0= & t_{\varepsilon} \int_{\mathbb{R}^{N}}\left(\left|\nabla \vartheta_{\varepsilon}\right|^{2}+\|V\|_{L^{\infty}(\Omega)} \vartheta_{\varepsilon}^{2}\right) d x+t_{\varepsilon}^{3} \int_{\mathbb{R}^{N}}\left|\nabla \vartheta_{\varepsilon}^{2}\right|^{2} d x \\
& -\lambda t_{\varepsilon} \int_{\mathbb{R}^{N}}\left|\vartheta_{\varepsilon}\right|^{q_{1}} d x-t_{\varepsilon}^{2\left(2^{*}\right)-1} .
\end{aligned}
$$

We claim that there is a positive constant $d>0$ such that $C_{\varepsilon}:=t_{\varepsilon}^{q_{1}} / q_{1} \geq$ $d>0, \forall \varepsilon>0$. Otherwise, we could find a sequence $\varepsilon_{n} \rightarrow 0$ such that $t_{\varepsilon_{n}} \rightarrow 0$. Up to a subsequence (still denote by $\varepsilon_{n}$ ), we have $t_{\varepsilon_{n}} \vartheta_{\varepsilon_{n}} \rightarrow 0$. Therefore, $0<c \leq \sup _{t>0} I\left(t_{\varepsilon_{n}} \vartheta_{\varepsilon_{n}}\right)=I(0)=0$, which is a contradiction. The claim is true. Next there are two cases to be considered: either $t_{\varepsilon} \leq 1$ or $t_{\varepsilon}>1$. For 
the case $t_{\varepsilon} \leq 1$, we have

$$
\begin{aligned}
& \frac{t_{\varepsilon}^{2}}{2} \int_{\mathbb{R}^{N}}\left(\left|\nabla \vartheta_{\varepsilon}\right|^{2}+\|V\|_{L^{\infty}(\Omega)} \vartheta_{\varepsilon}^{2}\right) d x-\frac{\lambda t_{\varepsilon}^{q_{1}}}{q_{1}} \int_{\mathbb{R}^{N}}\left|\vartheta_{\varepsilon}\right|^{q_{1}} d x \\
& \quad \leq \frac{1}{2} \int_{\mathbb{R}^{N}}\left(\left|\nabla \vartheta_{\varepsilon}\right|^{2}+\|V\|_{L^{\infty}(\Omega)} \vartheta_{\varepsilon}^{2}\right) d x-\lambda C_{\varepsilon} \int_{\mathbb{R}^{N}}\left|\vartheta_{\varepsilon}\right|^{q_{1}} d x .
\end{aligned}
$$

Note the following inequality:

$$
(a+b)^{\alpha} \leq a^{\alpha}+\alpha(a+b)^{\alpha+1} b, \quad \text { for all } a, b>0 \text { and } \alpha \geq 1 .
$$

Since the function $t \mapsto t^{4} \int_{\mathbb{R}^{N}}\left|\nabla \vartheta_{\varepsilon}^{2}\right|^{2} d x / 4-t^{2\left(2^{*}\right)} / 2\left(2^{*}\right)$ attains its maximum at $t_{0}:=\left(\int_{\mathbb{R}^{N}}\left|\nabla \vartheta_{\varepsilon}^{2}\right|^{2} d x\right)^{2\left(2^{*}\right) /\left(2\left(2^{*}-4\right)\right)}$, we conclude that

$$
\begin{aligned}
I\left(t_{\varepsilon} \vartheta_{\varepsilon}\right) \leq & \frac{t_{\varepsilon}^{4}}{4} \int_{\mathbb{R}^{N}}\left|\nabla \vartheta_{\varepsilon}^{2}\right|^{2} d x-\frac{t_{\varepsilon}^{2\left(2^{*}\right)}}{2\left(2^{*}\right)}+\frac{1}{2} \int_{\mathbb{R}^{N}}\left(\left|\nabla \vartheta_{\varepsilon}\right|^{2}+\|V\|_{L^{\infty}(\Omega)} \vartheta_{\varepsilon}^{2}\right) d x \\
& -\lambda C_{\varepsilon} \int_{\mathbb{R}^{N}}\left|\vartheta_{\varepsilon}\right|^{q_{1}} d x \\
\leq & \frac{1}{2 N} S^{N / 2}+O\left(\varepsilon^{(N-2) / 2}\right)+\frac{1}{2} \int_{\mathbb{R}^{N}}\left(\left|\nabla \vartheta_{\varepsilon}\right|^{2}+\|V\|_{L^{\infty}(\Omega)} \vartheta_{\varepsilon}^{2}\right) d x \\
& -\lambda C_{\varepsilon} \int_{\mathbb{R}^{N}}\left|\vartheta_{\varepsilon}\right|^{q_{1}} d x .
\end{aligned}
$$

For the case $t_{\varepsilon}>1$, by (2.7), we have

$$
\begin{aligned}
t_{\varepsilon} & \leq\left(\int_{\mathbb{R}^{N}}\left(\left|\nabla \vartheta_{\varepsilon}\right|^{2}+\|V\|_{L^{\infty}(\Omega)} \vartheta_{\varepsilon}^{2}\right) d x+\int_{\mathbb{R}^{N}}\left|\nabla \vartheta_{\varepsilon}^{2}\right|^{2} d x\right)^{1 /\left(2\left(2^{*}\right)-4\right)} \\
& \leq\left(2 \int_{\mathbb{R}^{N}}\left(\left|\nabla \vartheta_{\varepsilon}\right|^{2}+\|V\|_{L^{\infty}(\Omega)} \vartheta_{\varepsilon}^{2}\right) d x+\int_{\mathbb{R}^{N}}\left|\nabla \vartheta_{\varepsilon}^{2}\right|^{2} d x\right)^{1 /\left(2\left(2^{*}\right)-4\right)}:=t_{1} .
\end{aligned}
$$

Since the function $t \rightarrow\left(t^{4} t_{1}^{\left(2\left(2^{*}\right)-4\right)}\right) / 4-t^{2\left(2^{*}\right)} / 2\left(2^{*}\right)$ is increasing on $\left[0, t_{1}\right)$, we have,

$$
\begin{aligned}
I\left(t_{\varepsilon} \vartheta_{\varepsilon}\right) \leq & \frac{t_{\varepsilon}^{4}}{4} \int_{\mathbb{R}^{N}} 2\left(\left|\nabla \vartheta_{\varepsilon}\right|^{2}+\|V\|_{L^{\infty}(\Omega)} \vartheta_{\varepsilon}^{2}\right) d x+\frac{t_{\varepsilon}^{4}}{4} \int_{\mathbb{R}^{N}}\left|\nabla \vartheta_{\varepsilon}^{2}\right|^{2} d x \\
& -\frac{\lambda t_{\varepsilon}^{q_{1}}}{q_{1}} \int_{\mathbb{R}^{N}}\left|\vartheta_{\varepsilon}\right|^{q_{1}} d x-\frac{t_{\varepsilon}^{2\left(2^{*}\right)}}{2\left(2^{*}\right)} \\
\leq & {\left[\frac{t_{\varepsilon}^{4} t_{1}^{\left(2\left(2^{*}\right)-4\right)}}{4}-\frac{t_{\varepsilon}^{2\left(2^{*}\right)}}{2\left(2^{*}\right)}\right]-\lambda C_{\varepsilon} \int_{\mathbb{R}^{N}}\left|\vartheta_{\varepsilon}\right|^{q_{1}} d x } \\
\leq & \frac{1}{2 N} S^{N / 2}+O\left(\varepsilon^{(N-2) / 2}\right)-\lambda C_{\varepsilon} \int_{\mathbb{R}^{N}}\left|\vartheta_{\varepsilon}\right|^{q_{1}} d x .
\end{aligned}
$$

Therefore, combining (2.8) and (2.9), if can we show that for small $\varepsilon>0$ $O\left(\varepsilon^{(N-2) / 2}\right)+C \int_{\mathbb{R}^{N}}\left(\left|\nabla \vartheta_{\varepsilon}\right|^{2}+\|V\|_{L^{\infty}(\Omega)} \vartheta_{\varepsilon}^{2}\right) d x-\lambda C_{\varepsilon} \int_{\mathbb{R}^{N}}\left|\vartheta_{\varepsilon}\right|^{q_{1}} d x<0,(2.10)$ then we have $I\left(t_{\varepsilon} \vartheta_{\varepsilon}\right)<\frac{1}{2 N} S^{N / 2}$, which concludes the proposition. 
To show (2.10), we change the variables and obtain

$$
\begin{aligned}
\int_{B_{R}} & \left(\left|\nabla \vartheta_{\varepsilon}\right|^{2}+\|V\|_{L^{\infty}(\Omega)} \vartheta_{\varepsilon}^{2}\right) d x-\lambda C_{\varepsilon} \int_{B_{R}}\left|\vartheta_{\varepsilon}\right|^{q_{1}} d x \\
\leq & C \varepsilon^{\frac{N-2}{4}} \int_{B_{R}}\left[\frac{|x|^{2}}{\left(\varepsilon+|x|^{2}\right)^{(N+2) / 2}}+\frac{1}{\left(\varepsilon+|x|^{2}\right)^{(N-2) / 2}}\right] d x \\
& -\lambda C \varepsilon^{\frac{(N-2) q_{1}}{8}} \int_{B_{R}} \frac{1}{\left(\varepsilon+|x|^{2}\right)^{(N-2) q_{1} / 4}} d x \\
\leq & C N \omega_{N} \varepsilon^{\frac{N-2}{4}}\left[1+\ln \left(R \varepsilon^{-1 / 2}\right)\right] \\
& -\lambda C N \omega_{N} \varepsilon^{\frac{N}{2}-\frac{(N-2) q_{1}}{8}} \int_{0}^{R / \varepsilon^{1 / 2}} \frac{r^{N-1}}{\left(1+r^{2}\right)^{(N-2) q_{1} / 4}} d r .
\end{aligned}
$$

Furthermore, we also have

$$
\int_{B_{2 R} \backslash B_{R}}\left(\left|\nabla \vartheta_{\varepsilon}\right|^{2}+\|V\|_{L^{\infty}(\Omega)} \vartheta_{\varepsilon}^{2}\right) d x-\lambda C_{\varepsilon} \int_{B_{2 R} \backslash B_{R}}\left|\vartheta_{\varepsilon}\right|^{q_{1}} d x \leq C \varepsilon^{\frac{N-2}{4}} .
$$

Therefore, we choose some proper $\sigma>0$, whose value is to be determined later, such that

$$
\begin{aligned}
\lim _{\varepsilon \rightarrow 0} & {\left[O\left(\varepsilon^{(N-2) / 2}\right)+C \int_{\mathbb{R}^{N}}\left(\left|\nabla \vartheta_{\varepsilon}\right|^{2}+\|V\|_{L^{\infty}(\Omega)} \vartheta_{\varepsilon}^{2}\right) d x-\lambda C_{\varepsilon} \int_{\mathbb{R}^{N}}\left|\vartheta_{\varepsilon}\right|^{q_{1}} d x\right] } \\
\leq & \lim _{\varepsilon \rightarrow 0} \varepsilon^{(N-2) / 4-\sigma}\left\{C \varepsilon^{(N-2) / 4+\sigma}+C \varepsilon^{\sigma}\left[1+\ln \left(R \varepsilon^{-1 / 2}\right)\right]\right. \\
& \left.-\lambda C_{\varepsilon} N \omega_{N} \varepsilon^{N / 2-(N-2) q_{1} / 8-(N-2) / 4+\sigma} \int_{0}^{R / \varepsilon^{1 / 2}} \frac{r^{N-1}}{\left(1+r^{2}\right)^{(N-2) q_{1} / 4}} d r\right\} .
\end{aligned}
$$

For any $\sigma>0$, we have $\lim _{\varepsilon \rightarrow 0} \varepsilon^{\sigma} \ln \left(R \varepsilon^{-1 / 2}\right)=0$. Since $\sigma_{1}:=N / 2-(N-2) q_{1} / 8-$ $(N-2) / 4<0$, choose $\sigma=-\sigma_{1} / 2$, then we get $(2.10)$.

Lemma 2.7. Any $(P S)_{c}$ sequence $\left\{v_{n}\right\}$ of $J$ is bounded in $E$.

Proof. Note that

$$
J\left(v_{n}\right)=\frac{1}{2} \int_{\mathbb{R}^{N}}\left|\nabla v_{n}\right|^{2} d x+\frac{1}{2} \int_{\mathbb{R}^{N}} V(x) f^{2}\left(v_{n}\right) d x-\int_{\mathbb{R}^{N}} H\left(x, f\left(v_{n}\right)\right) d x \rightarrow c,
$$

and for any $\omega \in E$,

$$
\begin{aligned}
\left\langle J^{\prime}\left(v_{n}\right), \omega\right\rangle= & \int_{\mathbb{R}^{N}} \nabla v_{n} \nabla \omega d x+\int_{\mathbb{R}^{N}} V(x) f^{\prime}\left(v_{n}\right) f\left(v_{n}\right) \omega d x \\
& -\int_{\mathbb{R}^{N}} h\left(x, f\left(v_{n}\right)\right) f^{\prime}\left(v_{n}\right) \omega d x \rightarrow 0 .
\end{aligned}
$$

We choose $\omega=\omega_{n}=f\left(v_{n}\right) / f^{\prime}\left(v_{n}\right)$, we can deduce that

$$
|\nabla \omega|=\left(1+\frac{2 f^{2}\left(v_{n}\right)}{1+2 f^{2}\left(v_{n}\right)}\right)\left|\nabla v_{n}\right| \text {. }
$$


By $\left(h_{3}\right)$, we obtain,

$$
\begin{aligned}
J\left(v_{n}\right)-\frac{1}{\theta}\left\langle J^{\prime}\left(v_{n}\right), \omega_{n}\right\rangle= & \int_{\mathbb{R}^{N}}\left[\frac{1}{2}-\frac{1}{\theta}\left(1+\frac{2 f^{2}\left(v_{n}\right)}{1+2 f^{2}\left(v_{n}\right)}\right)\right]\left|\nabla v_{n}\right|^{2} d x \\
& +\left(\frac{1}{2}-\frac{1}{\theta}\right) \int_{\mathbb{R}^{N}} V(x) f^{2}\left(v_{n}\right) d x \\
& +\frac{1}{\theta} \int_{\mathbb{R}^{N}}\left[h\left(x, f\left(v_{n}\right)\right) f\left(v_{n}\right)-\theta H\left(x, f\left(v_{n}\right)\right)\right] d x \\
\geq & \frac{\theta-4}{2 \theta} \int_{\mathbb{R}^{N}}\left[\left|\nabla v_{n}\right|^{2}+\left(1-\frac{2(\theta-2)}{k(\theta-4)}\right) V(x) f^{2}\left(v_{n}\right)\right] d x
\end{aligned}
$$

which implies that $\int_{\mathbb{R}^{N}}\left(\left|\nabla v_{n}\right|^{2}+V(x) f^{2}\left(v_{n}\right)\right) d x \leq C$. We deduce that $\left\{v_{n}\right\}$ is bounded.

Lemma 2.8. There is a sequence $\left\{z_{n}\right\} \subset \mathbb{R}^{N}$ and $R>0, \beta>0$ such that

$$
\int_{B_{R}\left(z_{n}\right)} f^{2}\left(v_{n}\right) d x \geq \beta .
$$

Proof. Let $\left\{v_{n}\right\}$ be a $(P S)_{c}$ sequence. Suppose by a contradiction that the lemma does not hold. Then by Lemma 2.2 it follows that

$$
\int_{\mathbb{R}^{N}}\left|f\left(v_{n}\right)\right|^{q} d x=o(1), \quad 2<q<2\left(2^{*}\right),
$$

and then by $\left(g_{1}\right)$ and $\left(g_{2}\right)$,

$$
\int_{\mathbb{R}^{N}} G\left(f\left(v_{n}\right)\right) d x=\int_{\mathbb{R}^{N}} g\left(f\left(v_{n}\right)\right) f\left(v_{n}\right) d x=o(1) .
$$

By the definition of $h$, we have

$$
\begin{aligned}
\int_{\mathbb{R}^{N}} H\left(x, f\left(v_{n}\right)\right) d x \leq & \frac{1}{2\left(2^{*}\right)} \int_{\Omega \cup\left\{v_{n} \leq f^{-1}(a)\right\}} f^{2\left(2^{*}\right)}\left(v_{n}\right) d x \\
& +\frac{\alpha}{2 k} \int_{\Omega^{c} \cap\left\{v_{n}>f^{-1}(a)\right\}} f^{2}\left(v_{n}\right) d x+o(1)
\end{aligned}
$$

and

$$
\begin{aligned}
\int_{\mathbb{R}^{N}} h\left(x, f\left(v_{n}\right)\right) f\left(v_{n}\right) d x= & \int_{\Omega \cup\left\{v_{n} \leq f^{-1}(a)\right\}} f^{2\left(2^{*}\right)}\left(v_{n}\right) d x \\
& +\frac{\alpha}{k} \int_{\Omega^{c} \cap\left\{v_{n}>f^{-1}(a)\right\}} f^{2}\left(v_{n}\right) d x+o(1) .
\end{aligned}
$$

Then since $\left\langle J^{\prime}\left(v_{n}\right), f\left(v_{n}\right) / f^{\prime}\left(v_{n}\right)\right\rangle=o(1)$, we have

$$
\begin{aligned}
\int_{\mathbb{R}^{N}} & {\left[1+\frac{2 f^{2}\left(v_{n}\right)}{1+2 f^{2}\left(v_{n}\right)}\right]\left|\nabla v_{n}\right|^{2} d x+\int_{\mathbb{R}^{N}} V(x) f^{2}\left(v_{n}\right) d x } \\
- & \frac{\alpha}{k} \int_{\Omega^{c} \cap\left\{v_{n}>f^{-1}(a)\right\}} f^{2}\left(v_{n}\right) d x=\int_{\Omega \cup\left\{v_{n} \leq f^{-1}(a)\right\}} f^{2\left(2^{*}\right)}\left(v_{n}\right) d x+o(1) .
\end{aligned}
$$


Let $l \geq 0$ be such that

$$
\begin{aligned}
\int_{\mathbb{R}^{N}} & \left(1+\frac{2 f^{2}\left(v_{n}\right)}{1+2 f^{2}\left(v_{n}\right)}\right)\left|\nabla v_{n}\right|^{2} d x+\int_{\mathbb{R}^{N}} V(x) f^{2}\left(v_{n}\right) d x \\
& -\frac{\alpha}{k} \int_{\Omega^{c} \cap\left\{v_{n}>f^{-1}(a)\right\}} f^{2}\left(v_{n}\right) d x \\
& \rightarrow l .
\end{aligned}
$$

Then $l>0$. Otherwise, we have $v_{n} \rightarrow 0$ which contradicts $c>0$. So,

$$
\int_{\Omega \cup\left\{v_{n} \leq f^{-1}(a)\right\}} f^{2\left(2^{*}\right)}\left(v_{n}\right) d x \rightarrow l .
$$

Since

$$
\int_{\mathbb{R}^{N}} V(x) f^{2}\left(v_{n}\right) d x-\frac{\alpha}{k} \int_{\Omega^{c} \cap\left\{v_{n}>f^{-1}(a)\right\}} f^{2}\left(v_{n}\right) d x \geq 0,
$$

by the definition of the best constant $S$,

$$
\begin{aligned}
& S \leq \frac{\int_{\mathbb{R}^{N}}\left|\nabla f^{2}\left(v_{n}\right)\right|^{2} d x}{\left(\int_{\mathbb{R}^{N}}\left|f^{2}\left(v_{n}\right)\right|^{2^{*}} d x\right)^{2 / 2^{*}}} \\
& \leq \frac{\int_{\mathbb{R}^{N}}\left(1+\frac{2 f^{2}\left(v_{n}\right)}{1+2 f^{2}\left(v_{n}\right)}\right)\left|\nabla v_{n}\right|^{2} d x+\int_{\mathbb{R}^{N}} V(x) f^{2}\left(v_{n}\right) d x}{\left(\int_{\Omega \cup\left\{v_{n} \leq f^{-1}(a)\right\}} f^{2\left(2^{*}\right)}\left(v_{n}\right) d x\right)^{2 / 2^{*}}} \\
&-\frac{\alpha}{k} \int_{\Omega^{c} \cap\left\{v_{n}>f^{-1}(a)\right\}} f^{2}\left(v_{n}\right) d x \\
&\left.\rightarrow l^{1-2 / 2^{*}}, \quad \text { as } n \rightarrow \infty v_{n} \leq f^{-1}(a)\right\} \\
&\left.f^{2\left(2^{*}\right)}\left(v_{n}\right) d x\right)^{2 / 2^{*}}
\end{aligned}
$$

Therefore, $l \geq S^{\frac{N}{2}}$. It follows that

$$
\begin{aligned}
c= & \lim _{n \rightarrow \infty}\left[\frac{1}{2} \int_{\mathbb{R}^{N}}\left|\nabla v_{n}\right|^{2} d x+\frac{1}{2} \int_{\mathbb{R}^{N}} V(x) f^{2}\left(v_{n}\right) d x-\int_{\mathbb{R}^{N}} H\left(x, f\left(v_{n}\right)\right) d x\right] \\
\geq & \lim _{n \rightarrow \infty}\left[\frac{1}{4} \int_{\mathbb{R}^{N}}\left(1+\frac{2 f^{2}\left(v_{n}\right)}{1+2 f^{2}\left(v_{n}\right)}\right)\left|\nabla v_{n}\right|^{2} d x+\frac{1}{4} \int_{\mathbb{R}^{N}} V(x) f^{2}\left(v_{n}\right) d x\right. \\
& \left.-\frac{\alpha}{4 k} \int_{\Omega^{c} \cap\left\{v_{n}>f^{-1}(a)\right\}} f^{2}\left(v_{n}\right) d x-\frac{1}{2\left(2^{*}\right)} \int_{\Omega \cup\left\{v_{n} \leq f^{-1}(a)\right\}}\left(f\left(v_{n}\right)\right)^{2\left(2^{*}\right)} d x\right] \\
\rightarrow & \left(\frac{1}{4}-\frac{1}{2\left(2^{*}\right)}\right) l \\
\geq & \frac{1}{2 N} S^{\frac{N}{2}},
\end{aligned}
$$

which is a contradiction since $c<\frac{1}{2 N} S^{\frac{N}{2}}$. This completes the proof of the lemma. 
Lemma 2.9. The sequence $\left\{z_{n}\right\}$ is bounded in $\mathbb{R}^{N}$.

Proof. For each $R>0$ we set $\psi_{R} \in C_{0}^{\infty}(\mathbb{R},[0,1])$ such that $\psi_{R}(x)=0$ if $|x| \leq$ $R, \psi_{R}(x)=1$ if $|x| \geq 2 R$ and $\left|\nabla \psi^{\prime}(x)\right| \leq C R^{-1}$. Since $\left\langle J^{\prime}\left(v_{n}\right), \psi_{n} v_{n}\right\rangle=o(1)$, we have,

$$
\begin{aligned}
& \int_{\mathbb{R}^{N}}\left|\nabla v_{n}\right|^{2} \psi_{R} d x+\int_{\mathbb{R}^{N}} \nabla v_{n} \nabla \psi_{R} v_{n} d x+\int_{\mathbb{R}^{N}} V(x) f\left(v_{n}\right) f^{\prime}\left(v_{n}\right) \psi_{R} v_{n} d x \\
& -\int_{\mathbb{R}^{N}} h\left(x, f\left(v_{n}\right)\right) f^{\prime}\left(v_{n}\right) v_{n} \psi_{R} d x=o(1) .
\end{aligned}
$$

Taking $R$ large enough such that $\Omega \subset B_{R}(0)$, by $\left(g_{3}\right)$, we have,

$$
\begin{aligned}
& \int_{\mathbb{R}^{N}}\left|\nabla v_{n}\right|^{2} \psi_{R} d x+\int_{\mathbb{R}^{N}} \nabla v_{n} \nabla \psi_{R} v_{n} d x+\int_{\mathbb{R}^{N}} V(x) f\left(v_{n}\right) f^{\prime}\left(v_{n}\right) \psi_{R} v_{n} d x \\
& \quad \leq \frac{1}{k} \int_{\Omega^{c} \cup\left\{v_{n} \leq f^{-1}(a)\right\}} V(x) f^{\prime}\left(v_{n}\right) v_{n} \psi_{R} d x+o(1) .
\end{aligned}
$$

Then, by $\left(f_{6}\right)$, we have

$$
\begin{aligned}
& \left(\frac{1}{2}-\frac{1}{2 k}\right) \alpha \int_{\mathbb{R}^{N}} f^{2}\left(v_{n}\right) \psi_{R} d x \\
& \quad \leq \int_{\mathbb{R}^{N}}\left|\nabla v_{n}\right|^{2} \psi_{R} d x+\left(1-\frac{1}{k}\right) \int_{\mathbb{R}^{N}} V(x) f\left(v_{n}\right) f^{\prime}\left(v_{n}\right) v_{n} \psi_{R} d x \\
& \quad=-\int_{\mathbb{R}^{N}} \nabla v_{n} \nabla \psi_{R} v_{n} d x+o(1) \\
& \quad \leq \frac{C}{R}\left\|v_{n}\right\|_{2}\left\|\nabla v_{n}\right\|_{2}+o(1),
\end{aligned}
$$

which implies the result.

Lemma 2.10. If $v_{n} \rightarrow v$ in $E$, then $v$ is a nontrivial critical point of $J$.

Proof. Arguing as in [41], let $\varphi \in C_{0}^{\infty}\left(\mathbb{R}^{N}, \mathbb{R}\right)$ with $Q:=\operatorname{supp}(\varphi)$. Since $E \hookrightarrow$ $H^{1}\left(\mathbb{R}^{N}\right)$ is continuous, we see that

$$
\int_{\mathbb{R}^{N}} \nabla v_{n} \nabla \varphi d x \rightarrow \int_{\mathbb{R}^{N}} \nabla v \nabla \varphi d x .
$$

Besides, we also have $v_{n} \rightarrow v$ in $L_{l o c}^{p}\left(\mathbb{R}^{N}\right)$ for $p \in\left[2,2^{*}\right)$. Then, $v_{n} \rightarrow v$ a.e. on $Q$ as $n \rightarrow \infty$ and $\left|v_{n}(x)\right| \leq\left|\omega_{p}(x)\right|$ for every $n \in \mathbb{N}$ and a.e. on $Q$ with $w_{p}(x) \in L^{p}\left(\mathbb{R}^{N}\right)$ ( see e.g. Lemma A.1, [44]). Therefore,

$$
\begin{aligned}
& V(x) f\left(v_{n}\right) f^{\prime}\left(v_{n}\right) \varphi \rightarrow V(x) f(v) f^{\prime}(v) \varphi, \quad \text { a.e. on } Q, \text { as } n \rightarrow \infty, \\
& f^{2\left(2^{*}\right)-1}\left(v_{n}\right) f^{\prime}\left(v_{n}\right) \varphi \rightarrow f^{2\left(2^{*}\right)-1}(v) f^{\prime}(v) \varphi, \quad \text { a.e. on } Q, \text { as } n \rightarrow \infty, \\
& g\left(f\left(v_{n}\right)\right) f^{\prime}\left(v_{n}\right) \varphi \rightarrow g(f(v)) f^{\prime}(v) \varphi, \quad \text { a.e. on } Q, \text { as } n \rightarrow \infty
\end{aligned}
$$

Since

$$
\left|V(x) f\left(v_{n}\right) f^{\prime}\left(v_{n}\right) \varphi\right| \leq\left|V(x) f\left(v_{n}\right) \varphi\right| \leq \sup _{Q} V(x)\left|\omega_{2}\right||\varphi|
$$


the Lebesgue Dominated Convergence Theorem implies that

$$
\int_{\mathbb{R}^{N}} V(x) f\left(v_{n}\right) f^{\prime}\left(v_{n}\right) \varphi d x \rightarrow \int_{\mathbb{R}^{N}} V(x) f(v) f^{\prime}(v) \varphi d x
$$

Similarly, since $\left|f^{2\left(2^{*}\right)-1}\left(v_{n}\right) f^{\prime}\left(v_{n}\right) \varphi\right| \leq C\left|\omega_{2^{*}-1}\right|^{2^{*}-1}|\varphi|$, we have

$$
\int_{\mathbb{R}^{N}} f^{2\left(2^{*}\right)-1}\left(v_{n}\right) f^{\prime}\left(v_{n}\right) \varphi d x \rightarrow \int_{\mathbb{R}^{N}} f^{2\left(2^{*}\right)-1}(v) f^{\prime}(v) \varphi d x .
$$

For $\left|v_{n}\right| \leq 1$, by $\left(g_{1}\right)$ and $\left(g_{2}\right)$, we have

$$
\begin{aligned}
\left|g\left(f\left(v_{n}\right)\right) f^{\prime}\left(v_{n}\right) \varphi\right| & \leq \varepsilon\left|f\left(v_{n}\right)\right||\varphi|+C_{\varepsilon}\left|f\left(v_{n}\right)\right|^{q_{2}-1}|\varphi| \\
& \leq c\left(\varepsilon+C_{\varepsilon}\right)|\varphi|,
\end{aligned}
$$

and for $\left|v_{n}\right|>1$,

$$
\begin{aligned}
\left|g\left(f\left(v_{n}\right)\right) f^{\prime}\left(v_{n}\right) \varphi\right| & \leq \varepsilon\left|f\left(v_{n}\right)\right||\varphi|+C_{\varepsilon}\left|f\left(v_{n}\right)\right|^{q_{2}-1}\left|f^{\prime}\left(v_{n}\right)\right||\varphi| \\
& \leq \varepsilon\left|f\left(v_{n}\right)\right||\varphi|+C_{\varepsilon}\left|f\left(v_{n}\right)\right|^{q_{2}-1} \frac{\left|f\left(v_{n}\right)\right|}{\left|v_{n}\right|}|\varphi| \\
& \leq \varepsilon\left|\omega_{2}\right||\varphi|+C_{\varepsilon}\left|\omega_{2 *-1}\right|^{2^{*}-1}|\varphi| .
\end{aligned}
$$

Combining (2.11) and (2.12), the Lebesgue Dominated Convergence Theorem implies

$$
\int_{\mathbb{R}^{N}} g\left(f\left(v_{n}\right)\right) f^{\prime}\left(v_{n}\right) \varphi d x \rightarrow \int_{\mathbb{R}^{N}} g(f(v)) f^{\prime}(v) \varphi d x .
$$

Hence, $v$ is a critical point of $J$ because $C_{0}^{\infty}\left(\mathbb{R}^{N}\right)$ is dense in $E$. From Lemmas 2.8 and $2.9, v$ is nontrivial.

Lemma 2.11. Any critical point of $J$ is of class $C_{l o c}^{2, \alpha}\left(\mathbb{R}^{N}\right)$.

Proof. Let $v$ be a critical point of $J$. Then

$$
-\Delta v=w, \quad x \in \mathbb{R}^{N} \text {. }
$$

where $w:=f^{\prime}(v)[h(x, f(v)) f(v)-V(x) f(v)]$. By $\left(h_{1}\right),\left(h_{2}\right)$ and $|f(t)| \leq$ $2^{1 / 4}|t|^{1 / 2},\left|f(t) f^{\prime}(t)\right| \leq 1 / \sqrt{2}$ for all $t \in \mathbb{R}$, we have

$$
|w| \leq f^{\prime}(v)\left(C_{1}+C_{2}|f(v)|^{2\left(2^{*}\right)-1}\right) \leq C_{3}+C_{4}|v|^{\left(2\left(2^{*}\right)-2\right) / 2}
$$

in any ball $B_{R}$. Setting $p_{0}:=2\left(2^{*}\right) /\left(2\left(2^{*}\right)-2\right)>1$. As $v \in L^{2^{*}}\left(\mathbb{R}^{N}\right)$, we conclude that $w \in L^{p_{0}}\left(B_{R}\right)$. By the elliptic regularity theory, $w \in W^{2, p_{0}}\left(B_{R}\right)$. Using a standard bootstrap argument, we have $v \in W^{2, p}\left(B_{R}\right)$ for all $p \geq 2$ (see [23]). Hence, $v \in C_{l o c}^{1,1}\left(\mathbb{R}^{N}\right)$ and this implies that $w$ is locally Hölder continuous. Consequently, $v \in C_{l o c}^{2, \alpha}\left(\mathbb{R}^{N}\right)$ for some $\alpha \in(0,1)$.

Up to now, we have the following result:

Proposition 2.4. For all $\varepsilon>0$, there is a positive critical point $v_{\varepsilon} \in E$ associated to the functional

$$
J_{\varepsilon}(v)=\frac{1}{2} \int_{\mathbb{R}^{N}}\left(\varepsilon^{2}|\nabla v|^{2}+V(x) f^{2}(v)\right) d x-\int_{\mathbb{R}^{N}} H(x, f(v)) d x
$$

at the level $c_{\varepsilon}=\inf _{v \in E \backslash\{0\}} \max _{t \geq 0} J_{\varepsilon}(t v)>0$. 
Proof. Let $\left\{v_{n}\right\}$ be a $(P S)_{c}$ sequence such that $v_{n} \rightarrow v_{\varepsilon}$ in $E$. By the semicontinuity of norm, we have

$$
\int_{\mathbb{R}^{N}}\left|\nabla v_{\varepsilon}\right|^{2} d x \leq \lim _{n \rightarrow \infty} \int_{\mathbb{R}^{N}}\left|\nabla v_{n}\right|^{2} d x
$$

We show only the equality holds in (2.13). Otherwise, we have

$$
\begin{aligned}
c_{\varepsilon} \leq & J_{\varepsilon}\left(v_{\varepsilon}\right)-\frac{1}{\theta} J_{\varepsilon}^{\prime}\left(v_{\varepsilon}\right) v_{\varepsilon}=\left(\frac{1}{2}-\frac{1}{\theta}\right) \int_{\mathbb{R}^{N}}\left|\nabla v_{\varepsilon}\right|^{2} d x \\
= & \int_{\mathbb{R}^{N}}\left[\frac{1}{2} V(x) f^{2}\left(v_{\varepsilon}\right)-\frac{1}{\theta} V(x) f^{\prime}\left(v_{\varepsilon}\right) f\left(v_{\varepsilon}\right) v_{\varepsilon}\right] d x \\
& +\int_{\mathbb{R}^{N}}\left[\frac{1}{\theta} h\left(x, f\left(v_{\varepsilon}\right)\right) f^{\prime}\left(v_{\varepsilon}\right) v_{\varepsilon}-H\left(x, f\left(v_{\varepsilon}\right)\right)\right] d x \\
< & \lim _{n \rightarrow \infty}\left(\frac{1}{2}-\frac{1}{\theta}\right) \int_{\mathbb{R}^{N}}\left|\nabla v_{n}\right|^{2} d x \\
& +\lim _{n \rightarrow \infty} \int_{\mathbb{R}^{N}}\left[\frac{1}{2} V(x) f^{2}\left(v_{n}\right)-\frac{1}{\theta} V(x) f^{\prime}\left(v_{n}\right) f\left(v_{n}\right) v_{n}\right] d x \\
& +\lim _{n \rightarrow \infty} \int_{\mathbb{R}^{N}}\left[\frac{1}{\theta} h\left(x, f\left(v_{n}\right)\right) f^{\prime}\left(v_{n}\right) v_{n}-H\left(x, f\left(v_{n}\right)\right)\right] d x \\
= & \lim _{n \rightarrow \infty}\left[J_{\varepsilon}\left(v_{n}\right)-\frac{1}{2} J_{\varepsilon}^{\prime}\left(v_{n}\right) v_{n}\right]=c_{\varepsilon},
\end{aligned}
$$

which is a contradiction. Therefore, we have

$$
\lim _{n \rightarrow \infty} \int_{\mathbb{R}^{N}}\left|\nabla v_{n}\right|^{2} d x=\int_{\mathbb{R}^{N}}\left|\nabla v_{\varepsilon}\right|^{2} d x
$$

By a similar argument as Proposition 4.2 [18], we have

$$
\lim _{n \rightarrow \infty} \int_{\mathbb{R}^{N}} V(x) f^{2}\left(v_{n}\right) d x=\int_{\mathbb{R}^{N}} V(x) f^{2}\left(v_{\varepsilon}\right) d x .
$$

This together (2.14) and (4) in Proposition 2.4 [18] implies that $v_{n} \rightarrow v$ in $E$. We complete the proof.

\section{Proof of Theorem 1.1}

We suppose that $\partial \Omega$ is smooth and $0 \in \Omega$. Furthermore, without loss of generality, we assume

$$
V(0)=V_{0}:=\inf _{\Omega} V(x) .
$$

Denote by $J_{0}: E \rightarrow \mathbb{R}$ the functional given by

$$
J_{0}(v)=\frac{1}{2} \int_{\mathbb{R}^{N}}\left(\left|\nabla v_{n}\right|^{2}+V_{0} f^{2}(v)\right) d x-\int_{\mathbb{R}^{N}}\left(G(f(v))+\frac{1}{2\left(2^{*}\right)} f^{2\left(2^{*}\right)}(v)\right) d x,
$$

associated to the problem

$$
-\Delta v=f^{\prime}(v)\left(g(f(v))+f^{2\left(2^{*}\right)-1}(v)-V_{0} f(v)\right), \quad x \in \mathbb{R}^{N} .
$$


Under the conditions $\left(g_{1}\right)-\left(g_{4}\right)$, similar to the arguments of [41], (3.1) possesses a nontrivial solution $\omega$ at the level

$$
c_{0}=\inf _{v \in H^{1}\left(\mathbb{R}^{N}\right) \backslash\{0\}} \max _{t \geq 0} J_{0}(t v) .
$$

Moreover,

$$
0<c_{0}<\frac{1}{2 N} S^{N / 2}
$$

Let $J_{\varepsilon}: E \rightarrow \mathbb{R}$ denote the functional

$$
J_{\varepsilon}(v)=\frac{1}{2} \int_{\mathbb{R}^{N}}\left(|\nabla v|^{2}+V(\varepsilon x) f^{2}(v)\right) d x-\int_{\mathbb{R}^{N}} H(\varepsilon x, f(v)) d x
$$

associated to the equation

$$
-\Delta v=f^{\prime}(v)(h(\varepsilon x, f(v))-V(\varepsilon x) f(v)), \quad x \in \mathbb{R}^{N},
$$

and defined in the space

$$
E_{\varepsilon}=\left\{v \in H^{1}\left(\mathbb{R}^{N}\right): \int_{\mathbb{R}^{N}} V(\varepsilon x) f^{2}(v) d x<\infty\right\}
$$

equipped with the norm

$$
\|v\|_{\varepsilon}=\|\nabla v\|_{2}+\inf _{\xi>0} \frac{1}{\xi}\left[1+\int_{\mathbb{R}^{N}} V(\varepsilon x) f^{2}(\xi v) d x\right] .
$$

Let $\tilde{v}_{\varepsilon}(x)=v_{\varepsilon}(z), z=\varepsilon x$, be a critical point of $J_{\varepsilon}$ at the level

$$
b_{\varepsilon}=\inf _{v \in E_{\varepsilon} \backslash\{0\}} \max _{t \geq 0} J_{\varepsilon}(t v) .
$$

It is easy to check that $b_{\varepsilon}=\varepsilon^{-N} c_{\varepsilon}$. Furthermore, from Proposition 2.3, for each $\varepsilon>0$, we have $b_{\varepsilon}<\frac{1}{2 N} S^{N / 2}$. 
Lemma 3.1. $\lim _{\varepsilon \rightarrow 0} b_{\varepsilon} \leq c_{0}$.

Proof. We consider the function $\omega_{\varepsilon}=\phi(\varepsilon x) \omega(x)$, where $\phi \in C_{0}^{\infty}\left(\mathbb{R}^{N},[0,1]\right)$ is defined by

$$
\phi(x)= \begin{cases}1 & \text { if } x \in B_{\rho}, \\ 0 & \text { f } x \in \mathbb{R}^{N} \backslash B_{2 \rho},\end{cases}
$$

for some $\rho>0$. We will assume that $\bar{B}_{2 \rho} \subset \Omega$. It is easy to see that $\omega_{\varepsilon} \rightarrow \omega$ in $H^{1}\left(\mathbb{R}^{N}\right)$ as $\varepsilon \rightarrow 0$. Furthermore, $\operatorname{supp}\left(\omega_{\varepsilon}\right) \subset \Omega_{\varepsilon}:=\left\{x \in \mathbb{R}^{N}: \varepsilon x \in \Omega\right\}$ and

$$
\begin{aligned}
\int_{\mathbb{R}^{N}} V(\varepsilon x) f^{2}\left(\omega_{\varepsilon}\right) d x & =\int_{\operatorname{supp}\left(\omega_{\varepsilon}\right)} V(\varepsilon x) f^{2}\left(\omega_{\varepsilon}\right) d x \\
& \leq \int_{\Omega_{\varepsilon}} V(\varepsilon x) f^{2}\left(\omega_{\varepsilon}\right) d x \leq \sup _{\Omega} V \int_{\Omega_{\varepsilon}} \omega_{\varepsilon}^{2} d x \\
& \leq C \sup _{\Omega} V,
\end{aligned}
$$

which implies that $\omega_{\varepsilon} \in E_{\varepsilon}$. For each $\varepsilon>0$, let $t_{\varepsilon}$ satisfies $\max _{t \geq 0} J_{\varepsilon}\left(t \omega_{\varepsilon}\right)=$ $J_{\varepsilon}\left(t_{\varepsilon} \omega_{\varepsilon}\right)$. Then

$$
\begin{aligned}
b_{\varepsilon} \leq & \max _{t \geq 0} J_{\varepsilon}\left(t \omega_{\varepsilon}\right)=J_{\varepsilon}\left(t_{\varepsilon} \omega_{\varepsilon}\right) \\
\leq & \frac{t_{\varepsilon}^{2}}{2} \int_{\mathbb{R}^{N}}\left(\left|\nabla \omega_{\varepsilon}\right|^{2}+V(\varepsilon x) \omega_{\varepsilon}^{2}\right) d x-\int_{\mathbb{R}^{N}} H\left(\varepsilon x, f\left(t_{\varepsilon} \omega_{\varepsilon}\right)\right) d x \\
= & \frac{t_{\varepsilon}^{2}}{2} \int_{\mathbb{R}^{N}}\left(\left|\nabla \omega_{\varepsilon}\right|^{2}+V(\varepsilon x) \omega_{\varepsilon}^{2}\right) d x \\
& -\int_{\mathbb{R}^{N}}\left(G\left(f\left(t_{\varepsilon} \omega_{\varepsilon}\right)\right)+\frac{1}{2\left(2^{*}\right)} f^{2\left(2^{*}\right)}\left(t_{\varepsilon} \omega_{\varepsilon}\right)\right) d x .
\end{aligned}
$$

We claim that $t_{\varepsilon} \rightarrow 1$ as $\varepsilon \rightarrow 0$. First, we show that $t_{\varepsilon}$ is bounded. Otherwise, since $\left\langle J_{\varepsilon}^{\prime}\left(t_{\varepsilon} \omega_{\varepsilon}\right), t_{\varepsilon} \omega_{\varepsilon}\right\rangle=0$, we have

$$
\begin{aligned}
& \int_{\mathbb{R}^{N}} t_{\varepsilon}^{2}\left|\nabla \omega_{\varepsilon}\right|^{2} d x+\int_{\mathbb{R}^{N}} V(\varepsilon x) f\left(t_{\varepsilon} \omega_{\varepsilon}\right) f^{\prime}\left(t_{\varepsilon} \omega_{\varepsilon}\right) t_{\varepsilon} \omega_{\varepsilon} d x \\
& =\int_{\mathbb{R}^{N}}\left(g\left(f\left(t_{\varepsilon} v_{\varepsilon}\right)\right) f^{\prime}\left(t_{\varepsilon} \omega_{\varepsilon}\right) t_{\varepsilon} \omega_{\varepsilon}+f^{2\left(2^{*}\right)-1}\left(t_{\varepsilon} \omega_{\varepsilon}\right) f^{\prime}\left(t_{\varepsilon} \omega_{\varepsilon}\right) t_{\varepsilon} \omega_{\varepsilon}\right) d x .
\end{aligned}
$$

Then by (3.4),

$$
\begin{aligned}
\int_{\mathbb{R}^{N}}\left(\left|\nabla \omega_{\varepsilon}\right|^{2}+V(\varepsilon x) \omega_{\varepsilon}^{2}\right) d x & \geq \frac{1}{2} \int_{\mathbb{R}^{N}} \frac{f^{2\left(2^{*}\right)}\left(t_{\varepsilon} \omega_{\varepsilon}\right)}{t_{\varepsilon}^{2}} d x \\
& \geq \frac{1}{2}\left[\frac{f\left(t_{\varepsilon} \omega_{\varepsilon}\left(x_{0}\right)\right)}{t_{\varepsilon} \omega_{\varepsilon}\left(x_{0}\right)}\right]^{2} f^{2\left(2^{*}\right)-2}\left(t_{\varepsilon} \omega_{\varepsilon}\left(x_{0}\right)\right) \omega_{\varepsilon}^{2}\left(x_{0}\right)|\Omega| \\
& \rightarrow \infty,
\end{aligned}
$$

where $\omega_{\varepsilon}\left(x_{0}\right)=\min _{\bar{\Omega}} \omega_{\varepsilon}(x)>0$, which is a contradiction. Thus, up to a subsequence, we assume that $t_{\varepsilon} \rightarrow t_{1} \geq 0$. Moreover, by (3.3), we see that $t_{\varepsilon}^{2} \geq 2 b_{\varepsilon} / C$. Furthermore, we may assume, up to a sequence of $\varepsilon \rightarrow 0$, that 
$b_{\varepsilon} \geq c_{0}$. (otherwise, we are done). Therefore, $t_{\varepsilon}^{2} \geq 2 b_{\varepsilon} / C \geq 2 c_{0} / C>0$ and this implies that $t_{1}>0$. Passing to the limit in (3.4), we obtain

$$
\begin{aligned}
& t_{1}^{2} \int_{\mathbb{R}^{N}}|\nabla \omega|^{2} d x+V_{0} \int_{\mathbb{R}^{N}} f\left(t_{1} \omega\right) f^{\prime}\left(t_{1} \omega\right) t_{1} \omega d x \\
& \quad=\int_{\mathbb{R}^{N}} f^{2\left(2^{*}\right)-1}\left(t_{1} \omega\right) f^{\prime}\left(t_{1} \omega\right) t_{1} \omega d x+\int_{\mathbb{R}^{N}} g\left(f\left(t_{1} \omega\right)\right) f^{\prime}\left(t_{1} \omega\right) t_{1} \omega d x
\end{aligned}
$$

On the other hand, since

$$
\begin{aligned}
& \int_{\mathbb{R}^{N}}|\nabla \omega|^{2} d x+V_{0} \int_{\mathbb{R}^{N}} f(\omega) f^{\prime}(\omega) \omega d x \\
& =\int_{\mathbb{R}^{N}} f^{2\left(2^{*}\right)-1}(\omega) f^{\prime}(\omega) \omega d x+\int_{\mathbb{R}^{N}} g(f(\omega)) f^{\prime}(\omega) \omega d x
\end{aligned}
$$

then

$$
\begin{aligned}
V_{0} & \int_{\mathbb{R}^{N}}\left[\frac{f\left(t_{1} \omega\right) f^{\prime}\left(t_{1} \omega\right)}{t_{1} \omega}-\frac{f(\omega) f^{\prime}(\omega)}{\omega}\right] \omega^{2} d x \\
= & \int_{\mathbb{R}^{N}}\left[\frac{f^{2\left(2^{*}\right)-1}\left(t_{1} \omega\right) f^{\prime}\left(t_{1} \omega\right)}{t_{1} \omega}-\frac{f^{2\left(2^{*}\right)-1}(\omega) f^{\prime}(\omega)}{\omega}\right] \omega^{2} d x \\
& +\int_{\mathbb{R}^{N}}\left[\frac{g\left(f\left(t_{1} \omega\right)\right) f^{\prime}\left(t_{1} \omega\right)}{t_{1} \omega}-\frac{g(f(\omega)) f^{\prime}(\omega)}{\omega}\right] \omega^{2} d x .
\end{aligned}
$$

By $\left(g_{4}\right)$ and Lemma 2.1 , it is easy to see that

$$
L_{1}(s)=\frac{f^{2\left(2^{*}\right)-1}(s) f^{\prime}(s)-g(f(s)) f^{\prime}(s)}{s} \quad \text { and } \quad L_{2}(s)=\frac{f(s) f^{\prime}(s)}{s}
$$

are increasing and decreasing for $s>0$ respectively. Hence, we must have $t_{1}=1$. Moreover,

$$
J_{\varepsilon}\left(t_{\varepsilon} \omega_{\varepsilon}\right)=J_{0}\left(t_{\varepsilon} \omega_{\varepsilon}\right)+\frac{1}{2} \int_{\mathbb{R}^{N}}\left(V(\varepsilon x)-V_{0}\right) f^{2}\left(t_{\varepsilon} \omega_{\varepsilon}\right) d x .
$$

Taking the limit as $\varepsilon \rightarrow 0$ and using the definition of $\omega_{\varepsilon}$ and the Lebesgue Dominated Convergence Theorem, we conclude that

$$
\int_{\mathbb{R}^{N}}\left(V(\varepsilon x)-V_{0}\right) f^{2}\left(t_{\varepsilon} \omega_{\varepsilon}\right) d x \rightarrow 0 \quad \text { as } \varepsilon \rightarrow 0
$$

and therefore $\limsup _{\varepsilon \rightarrow 0} b_{\varepsilon} \leq \limsup _{\varepsilon \rightarrow 0} J\left(t_{\varepsilon} \omega_{\varepsilon}\right)=J(\omega)=c_{0}$.

Using similar arguments as that of Lemma 2.8, we have the following result.

Lemma 3.2. There are $\varepsilon_{0}>0$, a family $\left\{y_{\varepsilon}\right\} \subset \mathbb{R}^{N}$ and $R>0, \beta>0$ such that

$$
\int_{B_{R}\left(y_{\varepsilon}\right)} f^{2}\left(\tilde{v}_{\varepsilon}\right) d x \geq \beta \quad \text { for } 0<\varepsilon \leq \varepsilon_{0} .
$$


Lemma 3.3. $\left\{\varepsilon y_{\varepsilon}\right\}$ is bounded in $\mathbb{R}^{N}$. Moreover, $\operatorname{dist}\left(\varepsilon y_{\varepsilon}, \Omega\right) \leq \varepsilon R$.

Proof. We modify the arguments in [18]. For $\delta>0$, we define $K_{\delta}:=$ $\left\{x \in \mathbb{R}^{N}: \operatorname{dist}(x, \Omega) \leq \delta\right\}$. We consider the function $\phi_{\varepsilon}=\phi(\varepsilon x)$, where $\phi \in C^{\infty}\left(\mathbb{R}^{N},[0,1]\right)$ is defined by

$$
\phi(x)= \begin{cases}1 & \text { if } x \notin K_{\delta}, \\ 0 & \text { if } x \in \Omega,\end{cases}
$$

and $|\nabla \phi| \leq C \varepsilon \delta^{-1}$. Since $\left\langle J_{\varepsilon}^{\prime}\left(\tilde{v}_{\varepsilon}\right), \phi_{\varepsilon} \tilde{v}_{\varepsilon}\right\rangle=o(1)$, using $\left(g_{3}\right)$ and the fact that the support of $\phi_{\varepsilon}$ does not intercept $\Omega_{\varepsilon}$, we obtain

$$
\begin{aligned}
\alpha\left(\frac{1}{2}-\frac{1}{2 k}\right) \int_{\mathbb{R}^{N}} f^{2}\left(\tilde{v}_{\varepsilon}\right) \phi_{\varepsilon} d x & \leq \int_{\mathbb{R}^{N}}\left[\left|\nabla \tilde{v}_{\varepsilon}\right|^{2}+\left(V(\varepsilon x)-\frac{\alpha}{2 k}\right) f^{2}\left(\tilde{v}_{\varepsilon}\right)\right] \phi_{\varepsilon} d x \\
& =-\int_{\mathbb{R}^{N}} v_{\varepsilon} \nabla \tilde{v}_{\varepsilon} \nabla \phi_{\varepsilon} d x \\
& \leq C \delta^{-1} \varepsilon
\end{aligned}
$$

If for some sequence $\varepsilon_{n} \rightarrow 0$ we have

$$
B_{R}\left(y_{\varepsilon_{n}}\right) \cap\left\{x \in \mathbb{R}^{N}: \varepsilon_{n} x \in K_{\delta}\right\}=\emptyset,
$$

then $\int_{B_{R}\left(y_{\varepsilon_{n}}\right)} f^{2}\left(v_{\varepsilon_{n}}\right) d x \leq C \delta^{-1} \varepsilon_{n}$, which contradicts Lemma 3.2. Thus (3.5) does not hold, that is, for all $\varepsilon$ there is an $x$ such that $\varepsilon x \in K_{\delta}$ and $\left|x-y_{\varepsilon}\right| \leq R$. It is easy to verify that this implies $\operatorname{dist}\left(\varepsilon y_{\varepsilon}, \Omega\right) \leq \varepsilon R+\delta$. From this fact we conclude the proof.

Remark 3.1. From Lemma 3.3 we can suppose that the family $\left\{y_{\varepsilon}\right\}$, defined in Lemma 3.2, can be taken in such a way that $\varepsilon y_{\varepsilon} \in \Omega$ for all $\varepsilon \in\left(0, \varepsilon_{0}\right]$. Indeed, if not, we replace $y_{\varepsilon}$ by $\varepsilon^{-1} x_{\varepsilon}$, where $x_{\varepsilon}$ comes from Lemma 3.3, so that $\left|\varepsilon y_{\varepsilon}-x_{\varepsilon}\right| \leq \varepsilon R$. Observing that $\left|y_{\varepsilon}-x_{\varepsilon} / \varepsilon\right| \leq R$, we can replace $R$ by $2 R$ in Lemma 3.2.

Lemma 3.4. $\lim _{\varepsilon \rightarrow 0} V\left(\varepsilon y_{\varepsilon}\right)=V_{0}$. Moreover, $\omega_{\varepsilon}(x)=\tilde{v}_{\varepsilon}\left(x+y_{\varepsilon}\right)$ converges uniformly to a nontrivial solution of problem (3.1) over compact subsets of $\mathbb{R}^{N}$.

Proof. If $y_{n}:=y_{\varepsilon_{n}}$ are such that $\varepsilon_{n} y_{n} \rightarrow x_{0}$ as $\varepsilon_{n} \rightarrow 0$, we must prove that $V\left(x_{0}\right)=V_{0}$. By Lemma 3.3, we know that $x_{0} \in \bar{\Omega}$, that is, $V\left(x_{0}\right) \geq V_{0}$. Let us set $\tilde{v}_{n}(x):=\tilde{v}_{\varepsilon_{n}}(x), \omega_{n}(x):=\tilde{v}_{\varepsilon_{n}}\left(x+y_{n}\right)$. Then

$-\Delta \omega_{n}=f^{\prime}\left(\omega_{n}\right)\left(h\left(\varepsilon_{n} x+\varepsilon_{n} y_{n}, f\left(\omega_{n}\right)\right)-V\left(\varepsilon_{n} x+\varepsilon_{n} y_{n}\right) f\left(\omega_{n}\right)\right), \quad x \in \mathbb{R}^{N}$.

On the other hand, since $\left\|\omega_{n}\right\|_{H^{1}}=\left\|\tilde{v}_{n}\right\|_{H^{1}}$ is bounded, let $\omega \in H^{1}\left(\mathbb{R}^{N}\right)$ such that $\omega_{n} \rightarrow \omega$ in $H^{1}\left(\mathbb{R}^{N}\right)$. By Lemma 3.2, $\omega \geq 0, \omega \neq 0$. Denote $\chi(x)=$ $\lim _{n \rightarrow \infty} \chi_{\Omega}\left(\varepsilon_{n}+x+\varepsilon_{n} y_{n}\right)$ a.e. in $\mathbb{R}^{N}, \tilde{h}(x, f(\omega))=\chi(x)\left(f^{2(2 *)-1}(\omega)+g(f(\omega))\right)+$ $(1-\chi(x)) \tilde{g}(f(\omega))$. By (3.6), we have

$$
\begin{gathered}
\int_{\mathbb{R}^{N}}\left(\nabla \omega_{n} \nabla \phi+V\left(\varepsilon_{n} x+\varepsilon_{n} y_{n}\right) f\left(\omega_{n}\right) f^{\prime}\left(\omega_{n}\right) \phi\right) d x \\
=\int_{\mathbb{R}^{N}} h\left(\varepsilon_{n} x+\varepsilon_{n} y_{n}, f\left(\omega_{n}\right)\right) f^{\prime}\left(\omega_{n}\right) \phi d x,
\end{gathered}
$$


for all $\phi \in C_{0}^{\infty}\left(\mathbb{R}^{N}\right)$. Since $\left\|\tilde{v}_{n}\right\|_{\infty} \leq C$ for all $n$ (see e. g. [18]), by the Lebesgue Dominated Convergence Theorem we obtain

$$
\lim _{n \rightarrow \infty} \int_{\mathbb{R}^{N}} h\left(\varepsilon_{n} x+\varepsilon_{n} y_{n}, f\left(\omega_{n}\right)\right) f^{\prime}\left(\omega_{n}\right) \phi d x=\int_{\mathbb{R}^{N}} \tilde{h}(x, f(\omega)) f^{\prime}(\omega) \phi d x,
$$

for all $\phi \in C_{0}^{\infty}\left(\mathbb{R}^{N}\right)$. Taking the limit in (3.7) we see that $\omega$ satisfies

$$
\int_{\mathbb{R}^{N}}\left(\nabla \omega \nabla \phi+V\left(x_{0}\right) f(\omega) f^{\prime}(\omega) \phi\right) d x=\int_{\mathbb{R}^{N}} \tilde{h}(x, f(\omega)) f^{\prime}(\omega) \phi d x,
$$

for all $\phi \in C_{0}^{\infty}\left(\mathbb{R}^{N}\right)$. Therefore, $\omega$ is a critical point of the functional

$$
\mathcal{F}(v)=\frac{1}{2} \int_{\mathbb{R}^{N}}\left(|\nabla v|^{2}+V\left(x_{0}\right) f^{2}(v)\right) d x-\int_{\mathbb{R}^{N}} \tilde{H}(x, f(v)) d x,
$$

where $\tilde{H}(s)=\int_{0}^{s} \tilde{h}(x, t) d t$. If $x_{0} \in \Omega$, we have $\varepsilon_{n} x+\varepsilon_{n} y_{n} \in \Omega$ as $n \rightarrow \infty$. Hence, $\chi(x)=1$ for all $x \in \mathbb{R}^{N}$ and so $\omega$ is a critical point of the following functional

$\tilde{J}_{x_{0}}(v)=\frac{1}{2} \int_{\mathbb{R}^{N}}\left(|\nabla v|^{2}+V\left(x_{0}\right) f^{2}(v)\right) d x-\frac{1}{2\left(2^{*}\right)} \int_{\mathbb{R}^{N}} f^{2\left(2^{*}\right)}(v) d x-\int_{\mathbb{R}^{N}} \tilde{G}(f(v)) d x$.

Denoting by $c_{x_{0}}$ the mountain pass level associated to the functional $\tilde{J}_{x_{0}}$ and by $\tilde{c}$ the mountain pass level associated to the functional $\mathcal{F}$. Since $\tilde{H}(x, s) \leq$ $G(s)+s^{2\left(2^{*}\right)-1}$ for all $x \in \mathbb{R}^{N}$ and $s \in \mathbb{R}$, we obtain $\tilde{J}_{x_{0}}(v) \leq \mathcal{F}(v)$ for all $v \in$ $H^{1}\left(\mathbb{R}^{N}\right)$ and this implies that $c_{x_{0}} \leq \tilde{c}$. Set $A_{n}:=\left\{x \in R^{N}: \varepsilon_{n} x+\varepsilon_{n} y_{n} \in \Omega\right\}$ and

$$
\begin{aligned}
\Psi_{n}(x)= & \frac{\theta}{2} V\left(\varepsilon_{n} x+\varepsilon_{n} y_{n}\right) f^{2}\left(\omega_{n}(x)\right)-V\left(\varepsilon_{n} x+\varepsilon_{n} y_{n}\right) f\left(\omega_{n}(x)\right) f^{\prime}\left(\omega_{n}(x)\right) \omega_{n}(x) \\
& +h\left(\varepsilon_{n} x+\varepsilon_{n} y_{n}, f\left(\omega_{n}(x)\right)\right) f^{\prime}\left(\omega_{n}(x)\right) \omega_{n}(x) \\
& -\theta H\left(\varepsilon_{n} x+\varepsilon_{n} y_{n}, f\left(\omega_{n}(x)\right)\right) .
\end{aligned}
$$

If $x \in A_{n}$, using $\left(h_{3}\right)$ and $\left(g_{3}\right)$ we have

$$
\begin{aligned}
\Psi_{n}(x) \geq & \left(\frac{\theta}{2}-1\right) V\left(\varepsilon_{n} x+\varepsilon_{n} y_{n}\right) f^{2}\left(\omega_{n}(x)\right) \\
& +\frac{1}{2}\left(g\left(f\left(\omega_{n}(x)\right)-2 \theta G\left(f\left(\omega_{n}(x)\right)\right)\right) \geq 0 .\right.
\end{aligned}
$$

If $x \notin A_{n}$,

$$
\Psi_{n}(x) \geq\left(\frac{\theta}{2}-1-\frac{\theta}{2 k}\right) V\left(\varepsilon_{n} x+\varepsilon_{n} y_{n}\right) f^{2}\left(\omega_{n}(x)\right) \geq 0 .
$$

Since $c_{0} \leq c_{x_{0}} \leq \tilde{c} \leq \mathcal{F}(\omega)$ in $\mathbb{R}^{N}$ and $\Psi_{n} \rightarrow \Psi$ a.e., where $\Psi(x):=\frac{\theta}{2} V\left(x_{0}\right) f^{2}(\omega)-V\left(x_{0}\right) f(\omega) f^{\prime}(\omega) \omega+h(x, f(\omega)) f^{\prime}(\omega) \omega-\theta H(x, f(\omega))$. 
By the Fatou's Lemma and semicontinuity of norm, we obtain

$$
\begin{aligned}
\theta c_{0} \leq & \theta \mathcal{F}(\omega)=\theta \mathcal{F}(\omega)-\left\langle\mathcal{F}^{\prime}(\omega), \omega\right\rangle \\
= & \left(\frac{\theta}{2}-1\right) \int_{\mathbb{R}^{N}}|\nabla \omega|^{2} d x+\int_{\mathbb{R}^{N}} \Psi(x) d x \\
\leq & \liminf _{n \rightarrow \infty}\left(\frac{\theta}{2}-1\right) \int_{\mathbb{R}^{N}}\left|\nabla \omega_{n}\right|^{2} d x+\liminf _{n \rightarrow \infty} \int_{\mathbb{R}^{N}} \Psi_{n}(x) d x \\
= & \liminf _{n \rightarrow \infty}\left(\frac{\theta}{2}-1\right) \int_{\mathbb{R}^{N}}\left|\nabla \omega_{n}\right|^{2} d x+\liminf _{n \rightarrow \infty} \int_{\mathbb{R}^{N}}\left(\frac{\theta}{2} V\left(\varepsilon_{n} x\right) f^{2}\left(\tilde{v}_{n}\right)\right. \\
& -V\left(\varepsilon_{n} x\right) f\left(\tilde{v}_{n}\right) f^{\prime}\left(\tilde{v}_{n}\right) \tilde{v}_{n}+h\left(\varepsilon_{n} x, f\left(\tilde{v}_{n}\right) f^{\prime}\left(\tilde{v}_{n}\right) \tilde{v}_{n}\right. \\
& \left.\left.-\theta H\left(\varepsilon_{n} x, f\left(\tilde{v}_{n}\right)\right)\right)\right) d x \\
= & \liminf _{n \rightarrow \infty}\left(\theta J_{\varepsilon_{n}}\left(\tilde{v}_{n}\right)-\left\langle J_{\varepsilon_{n}}^{\prime}\left(\tilde{v}_{n}\right), \tilde{v}_{n}\right\rangle\right)=\theta \liminf _{n \rightarrow \infty} b_{\varepsilon} \leq \theta c_{0} .
\end{aligned}
$$

Thus, $\mathcal{F}(\omega)=c_{0}$ and $\lim _{\varepsilon \rightarrow 0} b_{\varepsilon}=c_{0}$. Moreover, if $V\left(x_{0}\right)>V_{0}$, by the fact that the dependence of the mountain pass value $c$ on the constant potential $V_{0}$ is continuous and increasing [34], we have $c_{0}<c_{x_{0}}=\tilde{c} \leq \mathcal{F}(\omega)=c_{0}$, which is a contradiction. Therefore, $V\left(x_{0}\right)=V_{0}$ and this implies that $x_{0} \in \Omega$ and $\mathcal{F}=\tilde{J}_{x_{0}}=J$. Hence, $\omega$ is a nontrivial solution of (3.1). Furthermore,

$$
-\Delta\left(\omega_{n}-\omega\right)=H_{n}, \quad x \in \mathbb{R}^{N},
$$

where

$$
\begin{aligned}
H_{n}(x)= & V_{0} f(\omega(x)) f^{\prime}(\omega(x))-V\left(\varepsilon_{n} x+\varepsilon_{n} y_{n}\right) f(\omega(x)) f^{\prime}(\omega(x)) \\
& +h\left(\varepsilon_{n} x+\varepsilon_{n} y_{n}, f(\omega(x))\right) f^{\prime}(\omega(x))-g(f(\omega(x))) f^{\prime}(\omega(x)) \\
& \left.-f^{2\left(2^{*}\right)-1}\right)(\omega(x)) f^{\prime}(\omega(x)) .
\end{aligned}
$$

As $\omega_{n} \rightarrow \omega$ a.e. in $\mathbb{R}^{N}$, this implies that $H_{n} \rightarrow 0$ a.e. in $\mathbb{R}^{N}$. Notice that for each compact subset $D$ in $\mathbb{R}^{N}$, we have $\left|H_{n}\right|,|\omega| \leq C$ since $\left\|\omega_{n}\right\|_{\infty} \leq C$ and $\left|\varepsilon_{n} x+\varepsilon_{n} y_{n}\right| \leq C$ for all $n$ and $x \in D$. Thus the Lebesgue Dominated Convergence Theorem implies that $H_{n} \rightarrow 0$ in $L_{\text {loc }}^{s}$ for all $s \geq 1$. By elliptic regularity theory [23], $\omega_{n} \rightarrow \omega$ in $C_{l o c}^{2}\left(\mathbb{R}^{N}\right)$ and the lemma is proved.

Using an similar argument as in Lemma 5.8 of [18], we have,

$$
\lim _{|x| \rightarrow \infty} \tilde{v}_{\varepsilon}(x)=0 .
$$

Thus, there exists an $R>0$ such that $\tilde{v}_{\varepsilon}(x)<a$ for all $|x| \geq R$. Choosing $\varepsilon_{0}>0$ sufficiently small such that $B_{R} \subset \Omega_{\varepsilon_{0}}$, since $\Omega_{\varepsilon}:=\left\{x \in \mathbb{R}^{N}: \varepsilon x \in \Omega\right\}$ and $\Omega$ is bounded, if $\varepsilon$ small enough, we deduce that $\Omega_{\varepsilon}$ large enough. So, $B_{R} \subset \Omega_{\varepsilon}$ can hold. We conclude that for all $\varepsilon \in\left(0, \varepsilon_{0}\right)$,

$$
-\Delta \tilde{v}_{\varepsilon}+V(\varepsilon x) f\left(\tilde{v}_{\varepsilon}\right) f^{\prime}\left(\tilde{v}_{\varepsilon}\right)=h\left(\tilde{v}_{\varepsilon}\right) f^{\prime}\left(\tilde{v}_{\varepsilon}\right), \quad x \in \mathbb{R}^{N} .
$$

Thus,

$$
-\varepsilon^{2} v_{\varepsilon}+V(x) f\left(v_{\varepsilon}\right) f^{\prime}\left(v_{\varepsilon}\right)=h\left(v_{\varepsilon}\right) f^{\prime}\left(v_{\varepsilon}\right), \quad x \in \mathbb{R}^{N},
$$

which implies that $u_{\varepsilon}=f\left(v_{\varepsilon}\right)$ is a positive solution of (1.4) for all $\varepsilon \in\left(0, \varepsilon_{0}\right)$.

Now, we show the concentration behavior of the solutions. By (3.8), $\omega_{\varepsilon}$ possesses a global maximum point $x_{\varepsilon} \in B_{\rho}$ for all $\varepsilon \in\left(0, \varepsilon_{0}\right)$ and some 
$\rho>0$. Considering the translation $\widetilde{\omega}_{\varepsilon}(x)=\omega_{\varepsilon}\left(x+x_{\varepsilon}\right)$, we may assume that $\omega_{\varepsilon}$ achieves its global maximum at the origin of $\mathbb{R}^{N}$. Using the fact that $\omega$ is spherically symmetric, $\partial \omega / \partial r<0$ for all $r>0$ and $\omega_{\varepsilon} \rightarrow \omega$ in $C_{l o c}^{2}\left(\mathbb{R}^{N}\right)$. By Lemma 4.2 in [34] we can conclude that $\omega_{\varepsilon}$ possesses no critical point other than the origin for all $\varepsilon \in\left(0, \varepsilon_{0}\right)$. Notice that the maximum value of $v_{\varepsilon}(z)=v_{\varepsilon}(\varepsilon x)=\tilde{v}_{\varepsilon}(x)=\omega_{\varepsilon}\left(x-y_{\varepsilon}\right)$ is achieved at the point $z_{\varepsilon}=\varepsilon y_{\varepsilon} \in \Omega$. As the function $f$ is strictly increasing, the maximum value of $u_{\varepsilon}(z)=f\left(v_{\varepsilon}(z)\right)$ is also achieved in this point. As $\nabla u_{\varepsilon}=f^{\prime}\left(v_{\varepsilon}\right) \nabla v_{\varepsilon}, u_{\varepsilon}$ possesses no critical point other $z_{\varepsilon}$. Finally, we show the exponential decay of solution. Since $\omega_{\varepsilon}(x) \rightarrow 0$ as $|x| \rightarrow+\infty$ and $f(t) / t \rightarrow 1$ as $t \rightarrow 0$, we have

$$
\lim _{|x| \rightarrow \infty} \frac{f\left(\omega_{\varepsilon}(x)\right) f^{\prime}\left(\omega_{\varepsilon}(x)\right)}{\omega_{\varepsilon}(x)}=1,
$$

and

$$
\lim _{|x| \rightarrow \infty} \frac{\left(g\left(f\left(\omega_{\varepsilon}(x)\right)\right) f\left(\omega_{\varepsilon}(x)\right)+f^{2\left(2^{*}\right)-1}\left(\omega_{\varepsilon}(x)\right)\right) f^{\prime}\left(\omega_{\varepsilon}(x)\right)}{\omega_{\varepsilon}(x)}=0 .
$$

Then, we can choose $R_{0}>0$ such that for all $\varepsilon \in\left(0, \varepsilon_{0}\right]$ and for all $|x| \geq R_{0}$,

$$
f\left(\omega_{\varepsilon}(x)\right) f^{\prime}\left(\omega_{\varepsilon}(x)\right) \geq \frac{3}{4} \omega_{\varepsilon}(x)
$$

and

$$
\left(g\left(f\left(\omega_{\varepsilon}(x)\right)\right) f\left(\omega_{\varepsilon}(x)\right)+f^{2\left(2^{*}\right)-1}\left(\omega_{\varepsilon}(x)\right)\right) f^{\prime}\left(\omega_{\varepsilon}(x)\right) \leq \frac{V_{0}}{2} \omega_{\varepsilon}(x) .
$$

Next, we shall apply the maximum principle (see [38]) to derive the properties of exponential decay of the bound state solution. Similar arguments have been used in $[1,18]$. We define $\psi(x)=M \exp (-\xi|x|)$, where $\xi$ and $M$ are such that $4 \xi^{2}<V_{0}$ and

$$
M \exp (-\xi|x|) \leq \xi^{2} \psi \quad \text { for all }|x|=R_{0} .
$$

It is easy to verify that

$$
\Delta \psi \leq \xi^{2} \psi \text { for all } x \neq 0 .
$$

Define $\psi_{\varepsilon}=\psi-\omega_{\varepsilon}$, then using (3.9), (3.10) and

$$
-\Delta \omega_{\varepsilon}+V\left(\varepsilon x+\varepsilon y_{\varepsilon}\right) f\left(\omega_{\varepsilon}\right) f^{\prime}\left(\omega_{\varepsilon}\right)=g\left(f\left(\omega_{\varepsilon}\right)\right) f\left(\omega_{\varepsilon}\right) f^{\prime}\left(\omega_{\varepsilon}\right)+\left(f\left(\omega_{\varepsilon}\right)\right)^{2\left(2^{*}\right)-1} f^{\prime}\left(\omega_{\varepsilon}\right)
$$

we obtain,

$$
\begin{aligned}
-\Delta \psi_{\varepsilon}+\frac{V_{0}}{4} \psi_{\varepsilon} \geq 0 & \text { in }|x| \geq R_{0}, \\
\psi_{\varepsilon} \geq 0 & \text { in }|x|=R_{0}, \\
\lim _{|x| \rightarrow \infty} \psi_{\varepsilon}(x)=0 . &
\end{aligned}
$$

Then, the maximum principle implies that $\psi_{\varepsilon} \geq 0$ for all $|x| \geq R_{0}$. Hence, $\psi_{\varepsilon}(x) \leq M \exp (-\xi|x|)$ for $|x| \geq R_{0}$ and $\varepsilon \in\left(0, \varepsilon_{0}\right]$. We then conclude that 


$$
u_{\varepsilon}(z)=f\left(v_{\varepsilon}(z)\right) \leq v_{\varepsilon}(z)=\tilde{v}\left(\frac{z}{\varepsilon}\right)=\omega_{\varepsilon}\left(\frac{z-z_{\varepsilon}}{\varepsilon}\right) \leq C \exp \left(-\xi\left|\frac{z-z_{\varepsilon}}{\varepsilon}\right|\right) .
$$

We have complete the proof of Theorem 1.1.

\section{References}

[1] Alves, C.O., do Ó, J.M., Souto, M.A.S.: Local mountain-pass for a class of elliptic problem in $\mathbb{R}^{N}$ involving critical exponents. Nonlinear Analysis TMA 46, 495-510 (2001)

[2] Ambrosetti, A., Badiale, M., Cingolani, S.: Semiclassical states of nonlinear Schrödinger equations. Arch. Ration. Mech. Anal. 140, 285-300 (1997)

[3] Ambrosetti, A., Malchiodi, A., Secchi, S.: Multiplicity results for some nonlinear Schrödinger equations with potentials. Arch. Ration. Mech. Anal. 159, 253-271 (2001)

[4] Amberosetti, A., Wang, Z.Q.: Positive solutions to a class of quasilinear elliptic equations on $\mathbb{R}$. Discrete Contin. Dyn. Syst. 9, 55-68 (2003)

[5] Berestycki, H., Lions, P.L.: Nonlinear scalar field equations I. Arch. Ration. Mech. Anal. 82, 313-346 (1983)

[6] Brezis, H., Nirenberg, L.: Positive solutions of nonlinear elliptic equations involving critical Sobolev exponents. Commun. Pure Appl. Math. 36, 437-477 (1983)

[7] Bonheure, D., Schaftingen, J.V.: Bound state solutions for a class of nonlinear Schrödinger equations. Rev. Mat. Iberoamericana 24(1), 297-351 (2008)

[8] Byeon, J., Jeanjean, L.: Standing waves for nonlinear Schrödinger equations with a general nonlinearity. Arch. Ration. Mech. Anal. 185, 185-200 (2007)

[9] Byeon, J., Wang, Z.Q.: Standing waves with a critical frequency for nonlinear Schrödinger equations II. Calc. Var. Partial Differ. Equ. 18, 207-219 (2003)

[10] Colin, M., Jeanjean, L.: Solutions for a quasilinear Schrödinger equations: a dual approach. Nonlinear Anal. TMA 56, 213-226 (2004)

[11] Dancer, N., Lam, K.Y., Yan, S.: The effect of the graph topology on the existence of multipeak solutions for nonlinear Schrödinger equations. Abstr. Appl. Anal. 3, 293-318 (1998)

[12] Dancer, N., Yan, S.: On the existence of multipeak solutions for nonlinear field equations on $\mathbb{R}^{N}$. Discrete Contin. Dyn. Syst. 6, 39-50 (2000)

[13] Del Pino, M., Felmer, P.L.: Semi-classical states for nonlinear Schrödinger equations. J. Funct. Anal. 149, 245-265 (1997)

[14] Del Pino, M., Felmer, P.L.: Multi-peak bound states for nonlinear Schrödinger equations. Ann. Inst. H. Poincaré Anal. Non Linéaire 15, 127-149 (1998) 
[15] Del Pino, M., Felmer, P.L.: Semi-classical states for nonlinear Schrödinger equations: a variational reduction method. Math. Ann. 324, 1-32 (2002)

[16] Del Pino, M., Felmer, P.L.: Local mountain passes for semilinear elliptic problems in unbounded domains. Calc. Var. Partial Differ. Equ. 4, 121-137 (1996)

[17] Del Pino, M., Felmer, P.: Multi-peak bound states for nonlinear Schrödinger equations. Ann. Inst. H. Poincaré Anal. Non Linéaire 15(2), 127-149 (1998)

[18] do Ó, J.M., Severo, U.: Solitary waves for a class of quasilinear Schrödinger quations in dimension two. Calc. Var. (2009). doi:10.1007/s00526-009-0286-6

[19] do Ó, J.M., Severo, U.: Quasilinear Schrödinger quations involving concave and convex nonlinrearities. Commun. Pure Appl. Anal. 8, 621-644 (2009)

[20] do Ó, J.M., Miyagaki, O.H., Soares, S.H.M.: Soliton solutions for quasilinear Schrödinger equations with critical growth. J. Differ. Equ. 248, 722-744 (2010)

[21] Floer, A., Weinstein, A.: Nonspreading wave packets for the cubic Schrödinger equation with a bounded potential. J. Funct. Anal. 69, 397-408 (1986)

[22] Gidas, B., Ni, W.M., Nirenberg, L.: Symmetry of positive solutions of nonlinear elliptic equations in $\mathbb{R}^{N}$. In: Nachbin, L. (ed.) Math. Analysis and Appl., Part A, Adv. Math. Suppl. Studies 7A. Academic Press, London, pp 369-402 (1981)

[23] Gilbarg, D., Trudinger, N.S.: Elliptic partical differential equation of second order. Springer, Berlin (1989)

[24] Jeanjean, L., Tanaka, K.: Singularly perturbed elliptic problems with superlinear or asymptotically linear nonlinearities. Calc. Var. Partial Differ. Equ. 21, 287-318 (2004)

[25] Kang, X., Wei, J.: On interacting bumps of semi-classical states of nonlinear Schrödinger equations. Adv. Differ. Equ. 5, 899-928 (2000)

[26] Kurihura, S.: Large amplitude quasi-solitons in superfluid films. J. Phys. Soc. Jpn. 50, 3262-3267 (1981)

[27] Li, Y.Y.: On a singularly perturbed elliptic equation. Adv. Differ. Equ. 2, 955-980 (1997)

[28] Liu, J.Q., Wang, Z.Q.: Soliton solutions for quasilinear Schrödinger equations. Proc. Am. Math. Soc. 131, 441-448 (2003)

[29] Liu, J.Q., Wang, Y.Q., Wang, Z.Q.: Soliton solutions to quasilinear Schrödinger equations II. J. Differ. Equ. 187, 473-493 (2003)

[30] Moameni, A.: Existence of soliton solutions for a quasilinear Schrödinger equation involving critical exponent in $\mathbb{R}^{N}$. J. Differ. Equ. 229, 570-587 (2006)

[31] Moameni, A.: On the existence of standing wave soultions to quasilinear Schrödinger equations. Nonlinearity 19, 937-957 (2006)

[32] Moameni, A.: On a class of periodic quasilinear Schrödinger equations involving critical growth in $\mathbb{R}^{2}$. J. Math. Anal. Appl. 334, 775-786 (2007) 
[33] Nakamura, A.: Damping and modification of exciton solitary waves. J. Phys. Soc. Jpn. 42, 1824-1835 (1977)

[34] Ni, W.M., Takagi, I.: On the shap and the location of least-energy solutions to semilinear Neumann problem. Commun. Pure Appl. Math. 44, 819-851 (1991)

[35] Oh, Y.G.: Existence of semiclassical bound states of nonlinear Schrödinger equations with potentials of the class $(V)_{a}$. Commun. Partial Differ. Equ. 13, 1499-1519 (1988)

[36] Oh, Y.G.: Stability of semiclassical bound states of nonlinear Schrödinger equations with potentials. Commun. Math. Phys. 121, 11-33 (1989)

[37] Poppenberg, M., Schmitt, K., Wang, Z.Q.: On the existence of solition solutions to quasilinear Schrödinger equations. Calc. Var. Partial Differ. Equ. 14, 329-344 (2002)

[38] Protter, M.H., Weinberger, H.F.: Maximum Principles in Differential Equations. Springer, Berlin (1984)

[39] Rabinowitz, P.H.: Minimax Methods in Critical Point Theory with Applications to Differential Equations. In: CBMS Regional Conf. Ser. in Math. No., vol. 65. Amer. Math. Soc., Providence (1986)

[40] Rabinowitz, P.H.: On a class of nonlinear Schrödinger equations. Z. Angew. Math. Phys. 43, 270-291 (1992)

[41] Silva, E.A., Vieira, G.F.: Quasilinear asymptotically periodic Schrödinger equations with critical growth. Calc. Var. 39, 1-33 (2010)

[42] Wang, X.: On concentration of positive bound states of nonlinear Schrödinger equations. Commun. Math. Phys. 153, 229-244 (1993)

[43] Wang, X.F., Zeng, B.: On Concentration of positive bound states of nonlinear Schrödinger equations with competing positive functions. SIAM J. Math. Anal. 28(3), 633-655 (1997)

[44] Willem, M.: Minimax Theorems. Birkhäuser, Boston (1996)

Youjun Wang and Wenming Zou

Department of Mathematical Sciences

Tsinghua University

Beijing 100084

China

e-mail: yjwang@math.tsinghua.edu.cn

Wenming Zou

e-mail:wzou@math.tsinghua.edu.cn

Received: 2 October 2010.

Accepted: 5 May 2011. 